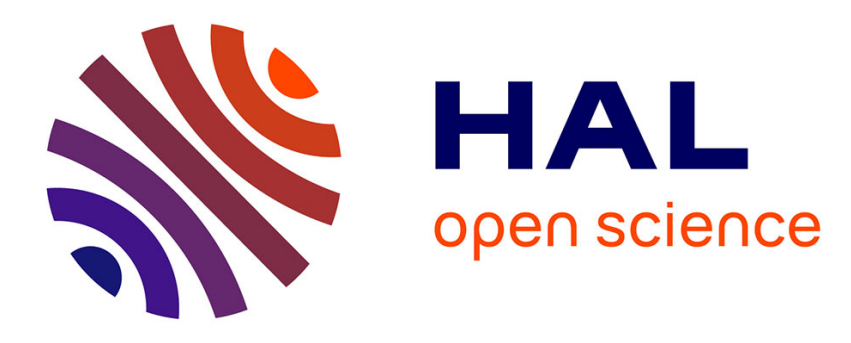

\title{
Local Lyapunov Exponents of the Quasi-Geostrophic Ocean Dynamics
}

Eugene Kazantsev

\section{To cite this version:}

Eugene Kazantsev. Local Lyapunov Exponents of the Quasi-Geostrophic Ocean Dynamics. [Research Report] RR-3074, INRIA. 1996, pp.45. inria-00073618

\section{HAL Id: inria-00073618 https://hal.inria.fr/inria-00073618}

Submitted on 24 May 2006

HAL is a multi-disciplinary open access archive for the deposit and dissemination of scientific research documents, whether they are published or not. The documents may come from teaching and research institutions in France or abroad, or from public or private research centers.
L'archive ouverte pluridisciplinaire HAL, est destinée au dépôt et à la diffusion de documents scientifiques de niveau recherche, publiés ou non, émanant des établissements d'enseignement et de recherche français ou étrangers, des laboratoires publics ou privés. 


\section{Local Lyapunov exponents of the quasi-geostrophic ocean dynamics.}

Evgueni Kazantsev

\section{$\mathrm{N}^{\circ} 3074$}

Décembre, 1996

THÈME 4 



\title{
Local Lyapunov exponents of the quasi-geostrophic ocean dynamics.
}

\author{
Evgueni Kazantsev \\ Thème 4 - Simulation et optimisation \\ de systèmes complexes \\ Projet NUMATH \\ Rapport de recherche $\mathrm{n}^{\circ} 3074$ - Décembre, 1996 - 45 pages
}

\begin{abstract}
The predictability of the quasi-geostrophic ocean model is considered in this paper. This is a simple dynamical model which assumes the ocean depth to be divided into $n$ layers of different water density. This model is driven by the wind stress on the surface, it includes effects of lateral and bottom friction, of the Earth rotation and the nonlinear interaction between adjacent layers.

Internal instability of the system leads to the rapid divergence of its trajectories limiting time of deterministic prediction of the system. To estimate the divergence rate of trajectories we compute Lyapunov exponents of this system. These exponents show us the average growth rate of a small possible perturbation of initial data on infinite time scale.

Along with Lyapunov exponents, their generalization, local Lyapunov exponents, are computed as a measure of divergence rate on finite time scales. These exponent provide us with the information about principal mechanisms of local instability. They can be useful in the forecasting of the forecast skill in meteorology and oceanology.

Numerous studies are performed in order to understand the influence of physical (form of the basin, number of layers) and numerical (grid resolution) parameters on the predictability of the system.

To study the geographical structure of the perturbation, the distribution of initial error with the fastest norm growth is distinguished. This distribution shows us the initial data structure the most sensible to errors and points out the regions where the most accuracy should be required. The structure of this function depends much on the time scale of prediction.
\end{abstract}

Key-words: Predictability, Local Lyapunov exponents, quasi geostrophic ocean model

(Résumé : tsvp)

Unité de recherche INRIA Lorraine

Technopôle de Nancy-Brabois, Campus scientifique, 615 rue de Jardin Botanique, BP 101, 54600 VILLERS LÈS NANCY (France)

Téléphone : (33) 83593030 - Télécopie : (33) 83278319

Antenne de Metz, technopôle de Metz 2000, 4 rue Marconi, 55070 METZ

Téléphone : (33) 87203500 - Télécopie : (33) 87763977 


\section{Exposants de Lyapunov locaux de la dynamique quasi-géostrophique de l'océan.}

Résumé : On considère la prédicibilité du modèle quasi-géostrophique de l'océan. C'est un modèle dynamique simple qui suppose la profondeur de l'océan séparée en $n$ couches avec différente densité de l'eau. Le modèle comprend le forçage du vent atmosphérique appliqué sur la couche de surface, la dissipation latérale et le frottement de fond, l'effet de la rotation de la Terre et l'interaction non linéaire entre les couches.

L'instabilité interne du système provoque la divergence rapide des trajectoires limitant le temps de prévision déterministe du système. Pour estimer la vitesse de divergence, on calcule les exposants de Lyapunov du modèle. Ces exposants mesurent la croissance moyenne d'une petite perturbation des données initiales en limite de temps infini.

On calcule aussi la généralisation des exposants de Lyapunov - les exposants locaux, qui mesurent la divergence pendant un temps fini. Ces exposants peuvent nous indiquer les mécanismes principaux de l'instabilité locale. Ils peuvent servir à prévoir la fiabilité des prévisions en météorologie et océanographie.

L'influence des paramètres physiques (forme du bassin, nombre des couches) et numériques (résolution du quadrillage) sur la prédicibilité du système a été étudié.

La distribution des données initiales dont la norme augmente le plus vite est distinguée pour étudier la structure géographique de la perturbation. Cette fonction représente la distribution des erreurs des donnés initiales susceptible de croître vite, donc elle nous indique les régions où il faut exiger le plus de précision. La structure de cette fonction dépend beaucoup de l'échelle de temps de la prévision.

Mots-clé : Prédicibilité, exposants de Lyapunov locaux, modèle quasi-géostrophique de l'océan. 


\section{Contents}

1 Introduction $\quad 3$

2 Model equations $\quad 5$

2.1 Quasi-geostrophic formulation . . . . . . . . . . . . . . . 5

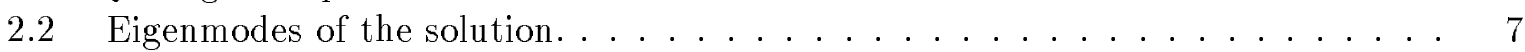

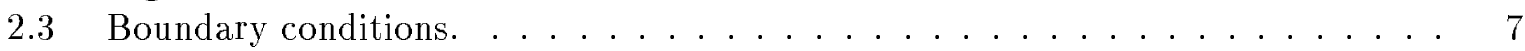

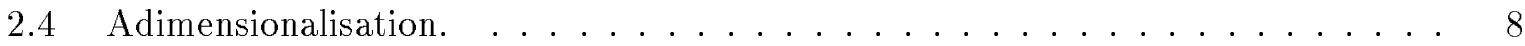

3 Evolution of a small perturbation of initial conditions. $\quad 9$

3.1 Variational formulation. . . . . . . . . . . . . . . . 11

3.2 Finite elements. . . . . . . . . . . . . . . . . . 11

3.3 Matricial formulation. . . . . . . . . . . . . . . . . 14

3.4 Evolution of the boundary constants. . . . . . . . . . . . 16

4 Lyapunov exponents of quasi-geostrophic circulation. $\quad \mathbf{2 0}$

4.1 Predictability characteristics on different time scales. . . . . . . . . . . . 20

4.2 Computation of Lyapunov Exponents. . . . . . . . . . . . . . . . . 24

5 Numerical results. $\quad \mathbf{2 5}$

5.1 Scheme of the integration in time. . . . . . . . . . . . . 27

5.2 One layer model in a square. . . . . . . . . . . . . . . . . 29

5.3 One layer model in a square. Higher resolution . . . . . . . . . . . . . . 33

5.4 Two layer model. . . . . . . . . . . . . . . . . 36

5.5 One layer model in the North Atlantic. . . . . . . . . . . . . . . 39

6 Conclusion $\quad 42$

\section{Introduction}

It has been known for 30 years that one cannot give a deterministic prediction of certain dynamical systems. One of the reasons of this phenomenon lies in the intrinsic instability of solutions of such systems. Nonlinear dynamical processes are the cause of the instability and due to this any error of initial data will tend to amplify with time even if the model is considered as perfect.

The internal instability of a system produces its chaotic behaviour. The concept of deterministic chaos introduced by Lorenz [Lorenz,63] is one of the most attractive paradigms for a nonlinear dynamical system. In this paradigm the temporal variation of the solution is considered to be a trajectory embedded in a strange attractor in phase space. One of the most striking features of the attractor of certain systems is that it has a finite dimension while the phase space of the system is infinite dimensional.

One can distinguish two complementary approaches to study its properties. The first of them concerns the study of the global features of the strange attractor. By this approach we can obtain the principal properties of the system like the existence of finite dimensional attractor of an infinite dimensional dynamical system [Temam,88], [Bernier,94]. By this approach one can

$\operatorname{RR} n^{\circ} 3074$ 
obtain the overestimates of its dimension. This has been made for systems related to geophysical flows (e.g. [Il'in,91]).

To evaluate its dimension numerically, there has been proposed by [Grassberger, Procaccia, 83] a method of reconstruction of the attractor of a system and calculation of its correlation dimension with a time series of some functional of the solution. However, time series required by this method to calculate the dimension of an attractor of a complex system are extremely long. It has been shown in [Ruelle,90] that the necessary length of time series is proportional to $10^{D}$, where $D$ is the attractor dimension. So it is very difficult to produce a time series required when the dimension exceeds 5-6, and one can never hope to calculate the attractor dimension of any real system. Nevertheless this method is applicable to simple systems providing a good possibility to calculate their attractor dimension.

For the complicate system which has an attractor of dimension larger than 6 , the KaplanYorke estimate seems to be much more robust when the Lyapunov exponents can be computed.

Another question related to the deterministic chaos is a limited predictability skill of its trajectory. This question is extremely important in studies of atmospheric and oceanic dynamics where it is related to the problem of weather predictions, one of the crucial problem of meteorology. Predictability studies are aimed at determining how far in advance and with what confidence we can predict the future state of a system when the present state is known withing a given uncertainty.

The Lyapunov exponents of the system are the well-known characteristics of long-time predictability. They measure the long-term average exponential rate of divergence (or convergence) of initially adjacent trajectories on an attractor, and thus quantify average predictability properties.

The second approach is to extract local characteristics of the strange attractor. It is well known that the behaviour of a solution can differ a lot on different parts of the attractor. In general, nearby trajectories need not diverge at the same rate on all parts of a chaotic attractor. The attractor inhomogenity leads to the phase spatial variability in the predictability time scale. This variability can result from the proximity of trajectories to unstable stationary point or limited loop and their stable and unstable manifold. This idea has been quantified in various ways by using local phase space information.

For atmospheric and oceanic dynamics the study of this kind of inhomogenity has been started by the work of [Charney, De Vore, 79], who showed that a system of atmospheric flows in a rotating channel with a spatially inhomogenous forcing can have several unstable equilibria. By using a truncated barotropic model of the atmosphere [Legras, Ghil,85] demonstrated that recurrent quasi stationary states occur in the vicinity of unstable stationary points in the phase space of the model. In [Dymnikov et al, 93] the mean time spent in the vicinity and the frequency of entering has been estimated by characteristics of unstable and stable manifolds of the stationary point respectively.

The temporal variation of the predictability is a central issue in the medium and extended range numerical weather prediction. Because forecast model shows considerable variability of the predictability, the prediction of the forecast skill gives important information to the numerical weather forecasts.

Numerous papers appeared last years are aimed at studying this variability and to produce a quality evaluation of a prediction along with prediction itself. Current literature usually refers to it as forecasting forecast skill. 
Several ways of study have been adopted for this purpose. One of the most effective is the forecast spread among an ensemble of forecasts. A truly Monte-Carlo simulation, however, is impracticable due to the huge number of degrees of freedom of real geophysical model. Thus, several techniques have been proposed to select the ensemble of initial conditions, forecast from different models, archived analysis, etc. For example, in [Molteni,Palmer,93] it has been proposed to use the fastest growing perturbation obtained from quasi-geostrophic model linearized about initial conditions of the operational model.

Parallel to these studies on operational models, much work has been done on low order minimal models of atmospheric and oceanic dynamics. These works provided the development of the theory of chaotic systems which is used as the guideline in the study of more complex models.

Along with variations of the predictability scale on the attractor, several recent studies have been devoted to the fact that the small initial error grows faster than the rate predicted by the largest Lyapunov exponent - a behaviour which is referred to as superexponential growth ([Lacarra,Talagrand,88], [Molteni,Palmer,93]). The study of this fact with simplest models ([Trevisan, 93],[Krishnamurty,93], [Nicolis et. al. 95]) revealed the generic mechanism that lies behind this phenomenon.

Effectively one can distinguish two principal reasons which have been proposed in literature. The first of them is the non-orthogonality of the eigenvectors of the linearized operator of the system, as a result of which certain linear combinations of eigenvectors can grow faster than perturbations along a particular eigendirection. And the second, the inhomogenity of the attractor resulting in the high variations of eigenvectors leads to enhanced short-time growth with respect to the long-time one.

These two reasons lead us to estimate the local predictability of a system by its local Lyapunov exponents introduced in [Abarbanel et al(1),91]. These exponents characterize the growth of a perturbation made at point after a finite time evolution by the dynamics. They depend on the particular trajectory (or its initial data) and on the period during which the integration is performed. When the integration time goes to infinity local exponents converge to the global ones. One of the issues addressed in this paper is the rate of this convergence.

As an example of the geophysical system we consider the quasi-geostrophic ocean dynamics. This is a rather simple model of the ocean circulation which was introduced by [Holland,78].

In section 2 the quasi-geostrophic formulation of the ocean model and its boundary conditions are briefly described. Section 3 is devoted to the development of the equation of evolution of a small perturbation to initial conditions of this model. In section 4 local Lyapunov exponents of the model are discussed. And the results of computation of these exponents are presented in section 5 .

\section{Model equations}

\subsection{Quasi-geostrophic formulation}

In this paper we consider ocean dynamics in the quasi-geostrophic formulation, i.e. we neglect all the thermodynamic effects. The vertical structure of the ocean is modelled by dividing the domain into $K$ layers of depth thickness $H_{k}$. The equation of the dynamics is written following

$\operatorname{RR} n^{\circ} 3074$ 
[Holland,78, Le Provost, Bernier, Blayo, 94]:

$$
\frac{\partial(\Delta \psi-\mathcal{W} \psi)}{\partial t}+J(\psi, \Delta \psi+f-\mathcal{W} \psi)=A \Delta^{2} \psi+F_{\text {wind }}-D_{b o t t o m},
$$

where $\psi=\psi_{k}(x, y), k=1, \ldots, K$ is the quasi-geostrophic streamfunction of $k$-th layer. Velocity fields for each layer can be calculated as

$$
u_{k}=-\frac{\partial \psi_{k}}{\partial x}, \quad v_{k}=\frac{\partial \psi_{k}}{\partial y} .
$$

Operators presented in the equation are Jacobian and Laplacian ones:

$$
J(\psi, \omega)=\left(\frac{\partial \psi}{\partial x} \frac{\partial \omega}{\partial y}-\frac{\partial \psi}{\partial y} \frac{\partial \omega}{\partial x}\right), \quad \Delta \psi=\frac{\partial^{2} \psi}{\partial x^{2}}+\frac{\partial^{2} \psi}{\partial y^{2}} .
$$

We assume the $\beta$-plane approximation for the Coriolis parameter $f$, which represents the effect of the Earth rotation in this equation, i.e. we suppose that this parameter is linear in $y$ coordinate:

$$
f=f_{0}+\beta\left(y-y_{0}\right)
$$

where $f_{0}$ and $y_{0}$ are the value of the Coriolis parameter and the coordinate of the mid-latitude of the basin. We suppose that this basin is located in the middle of North Atlantic, so we take the value of the Coriolis parameter in the middle of basin to be equal to $f_{0}=9.3 \times 10^{-5} \mathrm{~s}^{-1}$, and its meridional gradient $\beta=2 \times 10^{-11}(\mathrm{~m} \times s)^{-1}$.

The source of energy in this equation is presented by the atmospheric wind stress applied to the upper layer of the ocean. In this paper we take an approximation of the mean wind field over North Atlantic $\mathcal{F}(x, y)$ which will be discussed further:

$$
F_{\text {wind }}=-\tau_{0} \mathcal{F}(x, y) \delta_{k, 1},
$$

where the symbol $\delta_{i, j}=1$ for $i=j$, and $\delta_{i, j}=0$ otherwise, shows us that this term is not zero for the surface layer of the model only.

The dissipation in the equation (1) is composed by the harmonic lateral friction $A \Delta^{2} \psi$ and the bottom drag

$$
D_{\text {bottom }}=\varepsilon \Delta \psi \delta_{k, K}
$$

which is applied to the bottom layer only. Inter-layer interactions in this model are parametrized by the operator $\mathcal{W}$. We assume that interactions of adjacent layers are available only, so the matrix $\mathcal{W}$ is tridiagonal. Its structure can be represented as

$$
\mathcal{W}=\left(\begin{array}{cccc}
R_{1} & -R_{1} & \cdots & 0 \\
-R^{\prime}{ }_{2} & R_{2}+R^{\prime}{ }_{2} & -R_{2} & \cdots \\
\vdots & \ddots & \ddots & \ddots \\
0 & \cdots & -R_{K}^{\prime} & R_{K}^{\prime}
\end{array}\right)
$$

with

$$
R_{k}=\frac{f_{0}^{2} \rho_{0}}{g H_{k}\left(\rho_{k+1}-\rho_{k}\right)}, \quad R_{k}^{\prime}=\frac{f_{0}^{2} \rho_{0}}{g H_{k}\left(\rho_{k}-\rho_{k-1}\right)},
$$

where $\rho_{0}$ is the mean density of water, $\rho_{k}$ is the mean density in the $k$-th layer and $g$ is the gravity acceleration. 


\subsection{Eigenmodes of the solution.}

To avoid the necessity to solve an implicit problem with tridiagonal matrix $\mathcal{W}$ we shall use the fact that this matrix has a complete system of eigenvectors with nonnegative eigenvalues [Bengtsson, Temperton,79]. So there exists a matrix $\mathcal{B}$ such as

$$
\mathcal{B}^{-1} \mathcal{W B}=\Lambda
$$

where $\Lambda$ is a diagonal matrix of eigenvalues $\lambda_{k}, k=1, \ldots, K$ of $\mathcal{W}$. To determine the pair $\mathcal{B}, \mathcal{B}^{-1}$ exactly ([Bernier,90]) we require in addition $\sum_{k 1}^{K} \mathcal{B}_{k, k 1}^{-1}=\delta_{k, 1}$. We note, that so far $\sum_{j=1}^{K} \mathcal{W}_{i, j}=0, \forall i$, there exists an eigenvalue of this matrix which is equal to 0 . This eigenvalue we denote by $\Lambda_{1}=0$.

We denote by $\psi^{*}$ the streamfunction field in this eigenbasis: $\psi=\mathcal{B} \psi^{*}$. After multiplication by $\mathcal{B}^{-1}$ the equation (1) becomes:

$$
\frac{\partial\left(\Delta \psi^{*}-\Lambda \psi^{*}\right)}{\partial t}+\mathcal{B}^{-1} J(\psi, \Delta \psi+f-\mathcal{W} \psi)=A \Delta^{2} \psi^{*}+\mathcal{B}^{-1} F_{\text {wind }}-\mathcal{B}^{-1} D_{\text {bottom }} .
$$

We introduce the variable $\Theta^{*}=\Delta \psi^{*}-\Lambda \psi^{*}$ and we rewrite the friction terms in the eigenbasis. So far $\Delta \psi^{*}=\Theta^{*}+\Lambda \psi^{*}$, the lateral friction term can be expressed as

$$
A \Delta^{2} \psi^{*}=A\left(\Delta \Theta^{*}+\Lambda \Delta \psi^{*}\right)=A\left(\Delta \Theta^{*}+\Lambda \Theta^{*}+\Lambda^{2} \psi^{*}\right) .
$$

We can write the bottom drag term (5) as

$$
\begin{array}{r}
\mathcal{B}^{-1} D_{\text {bottom }}=\varepsilon \mathcal{B}^{-1} \Delta \psi \delta_{k, K}=\varepsilon \mathcal{B}^{-1}\left(\sum_{k 1} \mathcal{B}_{k, k 1}\left(\Theta_{k 1}^{*}+\Lambda_{k 1} \psi_{k 1}^{*}\right) \delta_{k, K}\right)= \\
\varepsilon\left(\sum_{k 1} \mathcal{B}_{k, K}^{-1} \mathcal{B}_{K, k 1}\left(\Theta_{k 1}^{*}+\Lambda \psi_{k 1}^{*}\right)\right)=\varepsilon \mathcal{V}\left(\Theta^{*}+\Lambda \psi^{*}\right)
\end{array}
$$

where

$$
\mathcal{V}_{k, k 1}=\mathcal{B}_{k, K}^{-1} \mathcal{B}_{K, k 1}
$$

So the equation (1) in the eigenbasis of the matrix $\mathcal{W}$ can be written as

$$
\frac{\partial \Theta^{*}}{\partial t}+\mathcal{B}^{-1} J(\psi, \Delta \psi+f-\mathcal{W} \psi)=A\left(\Delta \Theta^{*}+\Lambda \Theta^{*}+\Lambda^{2} \psi^{*}\right)-\varepsilon \mathcal{V}\left(\Theta^{*}+\Lambda \psi^{*}\right)+\mathcal{B}^{-1} F_{\text {wind }}
$$

\subsection{Boundary conditions.}

As it has been noted above we integrate the equation (11) in the domain $\Omega$. Following [Le Provost, Bernier, Blayo, 94] we take

$$
\psi_{k}^{*}=C_{k}(t) \quad \text { on the boundary of } \Omega \text { for } k=1, \ldots, K
$$

The value of the constant $C_{k}(t)$ will be calculated at each time step in order to have the mass conservation:

$$
\int \frac{\partial}{\partial t}\left(\psi_{k}^{*}\right) d x d y=0 \text { i.e. } \int \psi_{k}^{*}(t) d x d y=\int \psi_{k}^{*}(0) d x d y .
$$

For the mode which corresponds to the zero eigenvalue of matrix $\mathcal{W}$ we require boundary conditions

$\operatorname{RR} n^{\circ} 3074$

$$
\psi_{1}^{*}=0 \text { i.e. } C_{1}=0 \text { on the boundary. }
$$


Due to the harmonic lateral friction we have to take a complementary boundary conditions for the vorticity field. In frames of this work we choose the conditions

$$
\Delta \psi=0 \text { on the boundary }
$$

which are equivalent to the frequently used slip boundary condition.

\subsection{Adimensionalisation.}

As basic values characterizing the order of magnitudes of the main variables we take

- $L$ - the characteristic length of the domain,

- $U$ horizontal velocity scale obtained by the Sverdrup balance: $U=\frac{\tau_{0}}{\beta L H}$,

- $T=(\beta L)^{-1}$ characteristic time scale.

We introduce the dimensionless variables:

- $\psi^{\prime}=\frac{\psi}{L U}$, dimensionless streamfunction,

- $\Theta^{\prime}=\frac{L}{U} \Theta$, dimensionless potential vorticity,

- $\Lambda^{\prime}=L^{2} \Lambda$, dimensionless eigenvalue matrix,

- $t^{\prime}=\beta L t, x^{\prime}=\frac{x}{L}, y^{\prime}=\frac{y}{L}$, dimensionless time and geographic coordinates.

The adimensionalisation of the equation (1) gives us typical scales associated with the major processes described by this equation (see, for example, [Le Provost, Verron,87] for details):

- $\delta_{A}=A\left(\beta L^{3}\right)^{-1}$, width of the lateral friction boundary layer;

- $\delta_{\varepsilon}=\varepsilon(\beta L)^{-1}$, width of the bottom friction boundary layer;

- $\delta_{J}=U\left(\beta L^{3}\right)^{-1}$, width of the inertial boundary layer.

The dimensionless quasi-geostrophic equation (1) we write dropping the primes

$$
\begin{aligned}
\frac{\partial \Theta^{*}}{\partial t} & +\delta_{J} \mathcal{B}^{-1} J(\psi, \Delta \psi+\beta y-\mathcal{W} \psi)= \\
& =\delta_{A}\left(\Delta \Theta^{*}+\Lambda \Theta^{*}+\Lambda^{2} \psi^{*}\right)-\delta_{\varepsilon} \mathcal{V}\left(\Theta^{*}+\Lambda \psi^{*}\right)+\mathcal{B}^{-1} F_{w i n d} \\
\Theta^{*} & =\Delta \psi^{*}-\Lambda \psi^{*} \\
\psi & =\mathcal{B} \psi^{*}
\end{aligned}
$$

with boundary conditions

$$
\left\{\begin{array}{lll}
\Delta \psi_{k}=0 & \text { on } \partial \Omega & \text { for } k=1,2, \ldots, K \\
\psi_{1}^{*}=0 & \text { on } \partial \Omega & \\
\psi_{k}^{*}=C_{k}(t) & \text { on } \partial \Omega & \text { for } k=2,3, \ldots, K \\
\int_{\Omega} \psi_{k}^{*} d \Omega=\left.\int_{\Omega} \psi_{k}^{*}\right|_{t=0} d \Omega & & \text { for } k=2,3, \ldots, K .
\end{array}\right.
$$


and initial conditions

$$
\left.\psi^{*}\right|_{t=0}=\psi_{0}^{*} .
$$

The existence and uniqueness of the solution for the problem $(16),(17),(18)$ in appropriate functional spaces has been proved in [Bernier,90].

For simplicity we write the equation (16) as the system of equations of dynamics of each eigenmode $\Theta_{k}^{*}$ of the operator $\mathcal{W}$.

$$
\begin{aligned}
\frac{\partial \Theta_{k}^{*}}{\partial t} & +\delta_{J} \mathcal{B}^{-1} J(\psi, \Delta \psi+\beta y-\mathcal{W} \psi)= \\
& =\delta_{A}\left(\Delta \Theta_{k}^{*}+\Lambda_{k} \Theta_{k}^{*}+\Lambda_{k}^{2} \psi_{k}^{*}\right)-\delta_{\varepsilon} \mathcal{V}\left(\Theta^{*}+\Lambda \psi^{*}\right)+\mathcal{B}^{-1} F_{w i n d} \\
\Theta_{k}^{*} & =\Delta \psi_{k}^{*}-\Lambda_{k} \psi_{k}^{*}
\end{aligned}
$$

Here we drop the index " $k$ " for all terms composed of linear combination of eigenmodes. So we write the bottom friction term as $\mathcal{V}\left(\Theta^{*}+\Lambda \psi^{*}\right)$ assuming by that the $k$-th component of this product

$$
\left(\mathcal{V}\left(\Theta^{*}+\Lambda \psi^{*}\right)\right)_{k}=\sum_{k 1}^{K} \mathcal{V}_{k, k 1}\left(\Theta_{k 1}^{*}+\Lambda_{k 1} \psi_{k 1}^{*}\right) \text {. }
$$

The same remark concerns the products $(\mathcal{W} \psi)_{k}=\sum_{k 1}^{K} \mathcal{W}_{k, k 1} \psi_{k 1}$ and $\left(\mathcal{B}^{-1} \xi\right)_{k}=\sum_{k 1}^{K} \mathcal{B}_{k, k 1}^{-1} \xi_{k 1}$. We shall keep these designations in all the equations below.

\section{Evolution of a small perturbation of initial conditions.}

Let $\bar{\psi}(x, y, t), \bar{\Theta}(x, y, t)$ be a solution of the problem $((16),(17))$ with initial conditions

$$
\left.\bar{\psi}\right|_{t=0}=\bar{\psi}_{0}
$$

If we disturb the field $\bar{\psi}_{0}$ in $(21)$ by an error $\psi_{0}$ we obtain another solution $\psi^{\prime}(x, y, t)$ which corresponds to the initial conditions

$$
\left.\psi^{\prime}\right|_{t=0}=\psi_{0}^{\prime}=\bar{\psi}_{0}+\psi_{0}
$$

The evolution of the perturbation

$$
\psi(x, y, t)=\psi^{\prime}(x, y, t)-\bar{\psi}(x, y, t)
$$

will be described by the equations:

$$
\begin{aligned}
\frac{\partial \Theta_{k}^{*}}{\partial t} & +\delta_{J} \mathcal{B}^{-1} J(\bar{\psi}, \Delta \psi-\mathcal{W} \psi)+\delta_{J} \mathcal{B}^{-1} J(\psi, \Delta \bar{\psi}+\beta y-\mathcal{W} \bar{\psi})+ \\
& +\delta_{J} \mathcal{B}^{-1} J(\psi, \Delta \psi-\mathcal{W} \psi)=\delta_{A}\left(\Delta \Theta_{k}^{*}+\Lambda_{k} \Theta_{k}^{*}+\Lambda_{k}^{2} \psi_{k}^{*}\right)-\delta_{\varepsilon} \mathcal{V}\left(\Theta^{*}+\Lambda \psi^{*}\right) \\
\Theta_{k}^{*} & =\Delta \psi_{k}^{*}-\Lambda_{k} \psi_{k}^{*} \\
\psi & =\mathcal{B} \psi^{*}
\end{aligned}
$$

with initial conditions

RR $n^{\circ} 3074$

$$
\left.\psi\right|_{t=0}=\psi_{0}
$$


We suppose that our perturbation remains small during integration. We linearize the system (24) i.e. we neglect the term $\delta_{J} \mathcal{B}^{-1} J(\psi, \Delta \psi-\mathcal{W} \psi)$. The linear approximation of the evolution of a small error have a form

$$
\begin{aligned}
\frac{\partial \Theta_{k}^{*}}{\partial t} & +\delta_{J} \mathcal{B}^{-1} J(\bar{\psi}, \Delta \psi-\mathcal{W} \psi)+\delta_{J} \mathcal{B}^{-1} J(\psi, \Delta \bar{\psi}+\beta y-\mathcal{W} \bar{\psi})= \\
& =\delta_{A}\left(\Delta \Theta_{k}^{*}+\Lambda_{k} \Theta_{k}^{*}+\Lambda_{k}^{2} \psi_{k}^{*}\right)-\delta_{\varepsilon} \mathcal{V}\left(\Theta^{*}+\Lambda \psi^{*}\right), \\
\Theta_{k}^{*} & =\Delta \psi_{k}^{*}-\Lambda_{k} \psi_{k}^{*}, \\
\psi & =\mathcal{B} \psi^{*} .
\end{aligned}
$$

The boundary condition for the perturbation $\psi$ we can easily obtain from the boundary conditions for the solution (17) and the relationship (23)

$$
\left\{\begin{array}{lll}
\Delta \psi_{k}=0 & \text { on } \partial \Omega & \text { for } k=1,2, \ldots, K, \\
\psi_{1}^{*}=0 & \text { on } \partial \Omega & \\
\psi_{k}^{*}=C_{k}(t) & \text { on } \partial \Omega & \text { for } k=2,3, \ldots, K, \\
\int_{\Omega} \psi_{k}^{*} d \Omega=\left.\int_{\Omega} \psi_{k}^{*}\right|_{t=0} d \Omega & & \text { for } k=2,3, \ldots, K .
\end{array}\right.
$$

In order to solve equations (25) and to satisfy the boundary conditions (26), we introduce auxiliary functions

$$
\psi^{(0)}(x, y, t), \psi^{(1)}(x, y), \Theta^{(0)}(x, y, t), \Theta^{(1)}(x, y)
$$

so that

$$
\begin{array}{rlrl}
\psi_{k} & =\psi_{k}{ }^{(0)}(x, y, t)+C_{k}(t) \psi_{k}^{(1)}(x, y), & & \psi_{k}^{(0)}=0, \psi_{k}^{(1)}=1 \text { on } \partial \Omega, \\
\Theta_{k}=\Theta_{k}{ }^{(0)}(x, y, t)-\Lambda_{k} C_{k}(t) \Theta_{k}{ }^{(1)}(x, y), & \Theta_{k}{ }^{(0)}=0, \Theta_{k}{ }^{(1)}=1 \text { on } \partial \Omega,
\end{array}
$$

The values of $C_{k}(t)$ will be calculated as

$$
\begin{aligned}
C_{k}(t) & =\frac{\left.\int_{\Omega} \psi_{k}\right|_{t=0} d x d y-\int_{\Omega} \psi_{k}^{(0)}(x, y, t) d x d y}{\int_{\Omega} \psi_{k}^{(1)} d x d y} \\
C_{1} & =0
\end{aligned}
$$

If we represent functions in the form (27), the stationary equation of the system (25) will have the form

$$
\begin{aligned}
\Theta_{k}^{*} & =\Delta \psi_{k}^{*}-\Lambda_{k} \psi_{k}^{*} \\
\Theta_{k}{ }^{(0)}-\Lambda_{k} C_{k} \Theta_{k}^{(1)} & =\Delta \psi_{k}^{(0)}+C_{k} \Delta \psi_{k}^{(1)}-\Lambda_{k} \psi_{k}^{(0)}-C_{k} \Lambda_{k} \psi_{k}{ }^{(1)}
\end{aligned}
$$

Due to different boundary conditions for $\psi^{(0)}, \Theta^{(0)}$ and $\psi^{(1)}, \Theta^{(1)}$ we can split $(29)$ into

$$
\left\{\begin{aligned}
\Theta_{k}^{(0)} & =\Delta \psi_{k}^{(0)}-\Lambda_{k} \psi_{k}^{(0)} \\
-\Lambda_{k} \Theta_{k}^{(1)} & =\Delta \psi_{k}^{(1)}-\Lambda_{k} \psi_{k}^{(1)}
\end{aligned}\right.
$$

This couple of equations allows us to make the relationship between $\psi$ and $\Theta$ taking into account boundary conditions (26). 


\subsection{Variational formulation.}

In order to look for a weak solution of the problem (25), (26) we perform a variational formulation of this problem. We denote the $L_{2}$ scalar product by $\langle.,$.$\rangle :$

$$
<\psi, \varphi>=\iint_{\Omega} \psi \varphi d x d y
$$

Let us multiply the equation (25) by a test function $\varphi^{(0)}(x, y) \in H_{0}^{1}(\Omega)$ and perform the integration over the domain $\Omega$. Using the notation (31) we get

$$
\begin{array}{r}
<\frac{\partial \Theta_{k}^{*}}{\partial t}, \varphi^{(0)}>+\delta_{J}<\mathcal{B}^{-1} J(\bar{\psi}, \Delta \psi-\mathcal{W} \psi)+\mathcal{B}^{-1} J(\psi, \Delta \bar{\psi}+\beta y-\mathcal{W} \bar{\psi}), \varphi^{(0)}>= \\
=\delta_{A}\left(-<\nabla \Theta_{k}^{*}, \nabla \varphi^{(0)}>+<\Lambda_{k} \Theta_{k}^{*}+\Lambda_{k}^{2} \psi_{k}^{*}, \varphi^{(0)}>\right)-\delta_{\varepsilon}<\mathcal{V}\left(\Theta^{*}+\Lambda \psi^{*}\right), \varphi^{(0)}> \\
<\Theta_{k}^{*}, \varphi^{(0)}>=-<\nabla \psi_{k}^{*}, \nabla \varphi^{(0)}>-<\Lambda_{k} \psi_{k}^{*}, \varphi^{(0)}> \\
<\psi_{k}, \varphi^{(0)}>=<\mathcal{B} \psi^{*}, \varphi^{(0)}>
\end{array}
$$

Here we have used the Green formula:

$$
<\Delta \psi, \varphi^{(0)}>=-<\nabla \psi, \nabla \varphi^{(0)}>+\int_{\partial \Omega} \frac{\partial \psi}{\partial n} \varphi^{(0)} d s=-<\nabla \psi, \nabla \varphi^{(0)}>
$$

thanks to the fact that $\varphi^{(0)} \in H_{0}^{1}(\Omega)$, i.e. $\left.\varphi^{(0)}\right|_{\partial \Omega}=0$

Using representation $(27)$ of $\psi^{*}, \Theta^{*}$ we can rewrite $(32)$ as

$$
\begin{aligned}
<\frac{\partial \Theta_{k}^{*(0)}}{\partial t}, & \varphi^{(0)}>-\Lambda_{k}<\Theta_{k}^{(1)}, \varphi^{(0)}>\frac{\partial C_{k}(t)}{\partial t}+ \\
& +\delta_{J}<\mathcal{B}^{-1} J(\bar{\psi}, \Delta \psi-\mathcal{W} \psi), \varphi^{(0)}>+\delta_{J}<\mathcal{B}^{-1} J(\psi, \Delta \bar{\psi}+\beta y-\mathcal{W} \bar{\psi}), \varphi^{(0)}>= \\
= & \delta_{A}\left(-<\nabla \Theta_{k}^{*}, \nabla \varphi^{(0)}>+<\Lambda_{k} \Theta_{k}^{*}+\Lambda_{k}^{2} \psi_{k}^{*}, \varphi^{(0)}>\right)-\delta_{\varepsilon}<\mathcal{V}\left(\Theta^{*}+\Lambda \psi^{*}\right), \varphi^{(0)}>
\end{aligned}
$$

The couple of the stationary equations $(30)$ in the variational formulation has a form:

$$
\begin{aligned}
<\Theta_{k}^{*,(0)}, \varphi^{(0)}> & =-<\nabla \psi_{k}^{*,(0)}, \nabla \varphi^{(0)}>-<\Lambda_{k} \psi_{k}^{*,(0)}, \varphi^{(0)}> \\
-\Lambda_{k}<\Theta_{k}^{*,(1)}, \varphi^{(0)}> & =-<\nabla \psi_{k}^{*,(1)}, \nabla \varphi^{(0)}>-<\Lambda_{k} \psi^{*,(1)}, \varphi^{(0)}>.
\end{aligned}
$$

\section{$3.2 \quad$ Finite elements.}

The variational formulation (33), (34) of the problem (25), (26) allows us to look for a solution by the finite element method (FEM). The feasibility and utility of FEMs for modelling ocean dynamics was first addressed by [Fix,75]. He has stated that using FEMs brought numerous advantages of modelling like precision, natural conservation of model invariants, flexibility of discretisation of complex domains, etc. These advantages remain even when irregular discretisation of the domain is performed.

So far the solution produced by QG model of the North Atlantic typically includes a western boundary layer with intense velocity gradients, the advantage of refining the triangulation along the western boundary of the domain is rather clear. This helps to keep the quality of explicit eddy resolution by the model while working with lower number of grid nodes. The comparison of finite elements and finite difference models performed in [Le Provost, Bernier, Blayo, 94] revealed that the difference arose between simulations by FE and FD techniques can be judged as insignificant. $\mathrm{RR} \mathrm{n}^{\circ} 3074$ 
In spite of the fact that the number of operations per time step and grid node is much higher for FE model, the possibility of reducing the number of grid points considerably diminish the computational cost of a model run. The possibility to have a good precision working with a lower number of grid points is very valuable for Lyapunov exponents calculations due to very high number of operations per point.

Following [Bernier,90] we use $P 2$ finite elements, i.e. polynomials of second degree $p_{i}(x, y)=$ $a_{i} x^{2}+b_{i} x y+c_{i} y^{2}+d_{i} x+e_{i} y+f_{i}$. We cover our domain $\Omega$ by a set of non intersecting triangles and we define the set of integration points as the union of vertices and mi-edges of the triangles. We construct finite elements $p_{i}(x, y)$ so that being of the second order they are equal to 1 at the i-th integration point and zero at all other points. We enumerate the integration points beginning with internal points of the domain, and pushing all the boundary points to the end of the set. In other words we require

$$
\begin{aligned}
\left(x_{i}, y_{i}\right) \in \Omega \backslash \partial \Omega \text { for } i & =1, \ldots, N^{(0)}, \\
\left(x_{i}, y_{i}\right) \in \partial \Omega \text { for } i & =N^{(0)}+1, \ldots, N
\end{aligned}
$$

So far there are two types of boundary conditions in our problem (for $\psi^{(0)}, \Theta^{(0)}$ and $\psi^{(1)}, \Theta^{(1)}$ ) we need two systems of finite elements:

$$
\begin{array}{r}
p_{i}^{(0)}(x, y) \in H_{0}^{1}(\Omega), i=1, \ldots, N^{(0)} \\
p_{i}(x, y) \in H^{1}(\Omega), i=1, \ldots, N .
\end{array}
$$

The system $p_{i}^{(0)}(x, y)$ is composed of $N^{(0)}$ functions, all of them are equal to 0 on the boundary. The system $p_{i}(x, y)$ coincides with the system $p_{i}^{(0)}(x, y)$ for all inner points, but it contains also functions $\left(p_{i}(x, y) i=N^{(0)}+1, \ldots, N\right)$ which are equal to 1 at integration points on the boundary.

So far $\psi_{k}^{(0)}, \Theta_{k}^{(0)} \in H_{0}^{1}(\Omega)$, they can be expressed as linear combinations

$$
\psi_{k}^{(0)}(x, y, t)=\sum_{i=1}^{N^{(0)}} \psi_{i, k}^{(0)}(t) p_{i}^{(0)}(x, y), \quad \Theta_{k}^{(0)}(x, y, t)=\sum_{i=1}^{N^{(0)}} \Theta_{i, k}^{(0)}(t) p_{i}^{(0)}(x, y)
$$

Functions $\psi_{k}^{(1)}, \Theta_{k}^{(1)} \in H^{1}$ so they have to be presented by

$$
\psi_{k}^{(1)}(x, y)=\sum_{i=1}^{N} \psi_{i, k}^{(1)} p_{i}(x, y), \quad \Theta_{k}^{(1)}(x, y)=\sum_{i=1}^{N} \Theta_{i, k}^{(1)} p_{i}(x, y)
$$

Using these expressions we can write the discretized system (33), (34):

$$
\begin{aligned}
& \sum_{i} \frac{\partial \Theta_{i, k}^{*}(0)}{\partial t}<p_{i}^{(0)}, p_{j}^{(0)}>-\Lambda_{k} \frac{\partial C_{k}(t)}{\partial t} \sum_{i} \Theta_{i, k}^{(1)}<p_{i}, p_{j}^{(0)}>+ \\
+ & \delta_{J} \mathcal{B}^{-1} \sum_{i} \sum_{m} \bar{\psi}_{i}(\Delta \psi-\mathcal{W} \psi)_{m}<J\left(p_{i}, p_{m}\right), p_{j}^{(0)}>+ \\
+ & \delta_{J} \mathcal{B}^{-1} \sum_{i} \sum_{m} \psi_{i}(\Delta \bar{\psi}+\beta y-\mathcal{W} \bar{\psi})_{m}<J\left(p_{i}, p_{m}\right), p_{j}^{(0)}>=
\end{aligned}
$$




$$
\begin{aligned}
& =\delta_{A} \sum_{i}\left(-\Theta_{i, k}^{*}<\nabla p_{i}, \nabla p_{j}^{(0)}>+\left(\Lambda_{k} \Theta_{i, k}^{*}+\Lambda_{k}^{2} \psi_{i, k}^{*}\right)<p_{i}, p_{j}^{(0)}>\right)- \\
& -\delta_{\varepsilon} \mathcal{V} \sum_{i}\left(\Theta_{i}^{*}+\Lambda \psi_{i}^{*}\right)<p_{i}, p_{j}^{(0)}>
\end{aligned}
$$

and discretized stationary equations:

$$
\begin{aligned}
\sum_{i} \Theta_{i, k}^{*,(0)}<p_{i}^{(0)}, p_{j}^{(0)}> & =-\sum_{i} \psi_{i, k}^{*,(0)}<\nabla p_{i}^{(0)}, \nabla p_{j}^{(0)}>-\Lambda_{k} \sum_{i} \psi_{i, k}^{*,(0)}<p_{i}^{(0)}, p_{j}^{(0)}>, \\
-\Lambda_{k} \sum_{i} \Theta_{i, k}^{*,(1)}<p_{i}, p_{j}^{(0)}> & =-\sum_{i} \psi_{i, k}^{*,(1)}<\nabla p_{i}, \nabla p_{j}^{(0)}>-\Lambda_{k} \sum_{i} \psi_{i, k}^{*,(1)}<p_{i}, p_{j}^{(0)}>.
\end{aligned}
$$

To simplify notations we define matrices of mass and rigidity as

$$
\begin{array}{ll}
\mathcal{M}_{j, i}^{(0)}=\left\langle p_{i}^{(0)}, p_{j}^{(0)}\right\rangle, \quad \mathcal{C}_{j, i}^{(0)}=<\nabla p_{i}^{(0)}, \nabla p_{j}^{(0)}> & \begin{cases}i=1, \ldots, N^{(0)} \\
j=1, \ldots, N^{(0)}\end{cases} \\
\mathcal{M}_{j, i}^{(1 / 2)}=\left\langle p_{i}, p_{j}^{(0)}>, \quad \mathcal{C}_{j, i}^{(1 / 2)}=<\nabla p_{i}, \nabla p_{j}^{(0)}>\quad \begin{cases}i=1, \ldots, N \\
j=1, \ldots, N^{(0)}\end{cases} \right.
\end{array}
$$

Then matricial formulation of discretized equations will have a form:

$$
\begin{aligned}
\mathcal{M}^{(0)} \frac{\partial \Theta_{k}^{*(0)}}{\partial t} & -\Lambda_{k} \frac{\partial C_{k}(t)}{\partial t} \mathcal{M}^{(1 / 2)} \Theta_{k}^{(1)}= \\
= & -\sum_{m} \delta_{J} \mathcal{B}^{-1}\left(\sum_{i} \bar{\psi}_{i}<J\left(p_{i}, p_{m}\right), p_{j}^{(0)}>(\Delta \psi-\mathcal{W} \psi)_{m}\right)- \\
& -\sum_{i} \delta_{J} \mathcal{B}^{-1}\left(\sum_{m}(\Delta \bar{\psi}+\beta y-\mathcal{W} \bar{\psi})_{m}<J\left(p_{i}, p_{m}\right), p_{j}^{(0)}>\psi_{i}\right)+ \\
+\delta_{A}\left(-\mathcal{C}^{(1 / 2)} \Theta_{k}^{*}\right. & \left.+\Lambda_{k} \mathcal{M}^{(1 / 2)} \Theta_{k}^{*}+\Lambda_{k}^{2} \mathcal{M}^{(1 / 2)} \psi_{k}^{*}\right)-\delta_{\varepsilon} \mathcal{V}\left(\mathcal{M}^{(1 / 2)} \Theta^{*}+\Lambda \mathcal{M}^{(1 / 2)} \psi^{*}\right) \\
\mathcal{M}^{(0)} \Theta_{k}^{*,(0)} & =-\mathcal{C}^{(0)} \psi_{k}^{*,(0)}-\Lambda_{k} \mathcal{M}^{(0)} \psi_{k}^{*,(0)} \\
-\Lambda_{k} \mathcal{M}^{(1 / 2)} \Theta_{k}^{*,(1)} & =-\mathcal{C}^{(1 / 2)} \psi_{k}^{*,(1)}-\Lambda_{k} \mathcal{M}^{(1 / 2)} \psi_{k}^{*,(1)} \\
\psi & =\mathcal{B} \psi^{*}
\end{aligned}
$$

Here we have the possibility to choose $\Theta_{k}{ }^{(1)}$. We have only one condition which delimits our choice. This function must be equal to 1 on the boundary, but we can make an arbitrary choice of its values in the inner part of the domain. To facilitate numerical problems we can make this choice so, that

$$
\mathcal{M}^{(1 / 2)} \Theta_{k}^{(1)}=0, \quad \Theta_{i, k}^{(1)}=1 \text { for } i=N^{(0)}+1, \ldots, N
$$

In this case we do not need to calculate the value of time derivative of the boundary constant $C_{k}(t)$. Values of $\psi_{k}^{*,(1)}$ can be found as a solution of the equation (42):

$$
\begin{array}{r}
\left(\mathcal{C}^{(1 / 2)}+\Lambda_{k} \mathcal{M}^{(1 / 2)}\right) \psi_{k}^{*,(1)}=\Lambda_{k} \mathcal{M}^{(1 / 2)} \Theta_{k}^{*,(1)}=0 \\
\psi_{i, k}^{(1)}=1 \text { for } i=N^{(0)}+1, \ldots, N .
\end{array}
$$

$\operatorname{RR} \mathrm{n}^{\circ} 3074$ 
Adding to this equation the part (41) which concerns $\psi_{k}^{*,(0)}$ we obtain

$$
\left(\mathcal{C}^{(0)}+\Lambda_{k} \mathcal{M}^{(0)}\right) \psi_{k}^{*,(0)}+C_{k}(t)\left(\mathcal{C}^{(1 / 2)}+\Lambda_{k} \mathcal{M}^{(1 / 2)}\right) \psi_{k}^{*,(1)}=-\mathcal{M}^{(0)} \Theta_{k}^{*,(0)}+C_{k}(t) \Lambda_{k} \mathcal{M}^{(1 / 2)} \Theta_{k}^{*,(1)}
$$

So far matrices $\mathcal{C}^{(0)}, \mathcal{M}^{(0)}$ and $\mathcal{C}^{(1 / 2)}, \mathcal{M}^{(1 / 2)}$ are equal for $1 \leq i \leq N^{(0)}, 1 \leq j \leq N^{(0)}$ we can write

$$
\begin{array}{r}
-\left(\mathcal{C}^{(1 / 2)}+\Lambda_{k} \mathcal{M}^{(1 / 2)}\right) \psi_{k}^{*}=\mathcal{M}^{(1 / 2)} \Theta_{k}^{*}, \\
-\Lambda_{k} \psi_{i, k}^{*}=\Theta_{i, k}^{*}, \quad i=N^{(0)}+1, \ldots, N
\end{array}
$$

in other words we can write

$$
\psi_{k}^{*}=\mathcal{H} \Theta_{k}^{*},
$$

where $\mathcal{H}$ is a matrix of dimension $N \times N$ :

$$
\mathcal{H}=-\left\{\begin{array}{c|c|c}
\mathcal{C}^{(1 / 2)}+\Lambda_{k} \mathcal{M}^{(1 / 2)} \\
\hline 0 & -\Lambda_{k}
\end{array}\right\}^{-1} \times\left\{\begin{array}{l|l}
\multicolumn{2}{|c}{\mathcal{M}^{(1 / 2)}} \\
\hline 0 & 1
\end{array}\right\}
$$

It must be noted, that the matrix $\mathcal{H}$ is not a band-matrix. Being composed of the inversed $\mathcal{C}+\Lambda \mathcal{M}$ this matrix has a full structure. In the following we need to calculate products

$$
A=B \mathcal{H}
$$

with the matrix $\mathcal{H}$ on the right. So far $\mathcal{H}$ and $B$ are full matrices, the direct multiplication requires $2 \times N^{3}$ operations. Taking into account that $\mathcal{C}$ and $\mathcal{M}$ are band-matrices we can compute $A$ with $O\left(N^{5 / 2}\right)$ operations as

$$
A^{t}=\left(\mathcal{M}^{(1 / 2)}\right)^{t} \mathcal{R}, \text { where } \mathcal{R} \text { is a solution of }\left(\mathcal{C}^{(1 / 2)}\right)^{t} \mathcal{R}=B^{t} .
$$

The matrix $\mathcal{C}^{(1 / 2)}$ is a band-matrix, so we can find a column of $\mathcal{R}$ with $O\left(N^{3 / 2}\right)$ operations by Cholesky method. To find all $\mathcal{R}$ we need to solve $N$ linear systems, i.e. perform $N \times O\left(N^{3 / 2}\right)$ operations. The multiplication with the mass matrix $\mathcal{M}^{(1 / 2)}$ requires $O\left(N^{2}\right)$ operations only, because this matrix is a band-matrix also.

Further we write for simplicity just a product $B \mathcal{H}$ assuming that it is found by implicit method (50).

\subsection{Matricial formulation.}

We shall resolve the system (40) with respect to $\Theta^{*}$ in order to obtain the evolution of an error in a simple form $\frac{\partial \Theta^{*}}{\partial t}+\mathcal{G} \Theta^{*}=0$ where operator $\mathcal{G}$ depends on the basic trajectory $\bar{\psi}$.

First, we write the left hand side of (40) taking into account (44):

$$
\mathcal{M}^{(0)} \frac{\partial \Theta_{k}^{*(0)}}{\partial t}-\Lambda_{k} \frac{\partial C_{k}(t)}{\partial t} \mathcal{M}^{(1 / 2)} \Theta_{k}^{(1)}=\mathcal{M}^{(0)} \frac{\partial \Theta_{k}^{*(0)}}{\partial t}
$$

The right hand side of (40) is composed of 4 terms. The first two of them represent interactions between the basic solution $\bar{\psi}$ and the error. Using $J\left(p_{i}, p_{m}\right)=-J\left(p_{m}, p_{i}\right)$ and

$$
\Delta \psi-\mathcal{W} \psi=\mathcal{B B}^{-1}(\Delta \psi-\mathcal{W} \psi)=\mathcal{B}\left(\Delta \psi^{*}-\mathcal{B}^{-1} \mathcal{W B} \psi^{*}\right)=\mathcal{B}\left(\Delta \psi^{*}-\Lambda_{k} \psi^{*}\right)=\mathcal{B} \Theta^{*}
$$


we can write the first of them

$$
\begin{aligned}
& -\left(\mathcal{B}^{-1} \sum_{m}\left(\delta_{J} \sum_{i} \bar{\psi}_{i}<J\left(p_{i}, p_{m}\right), p_{j}^{(0)}>\right)(\Delta \psi-\mathcal{W} \psi)_{m}\right)_{k}= \\
& =\left(\mathcal{B}^{-1} \sum_{i}\left(\delta_{J} \sum_{m} \bar{\psi}_{m}<J\left(p_{i}, p_{m}\right), p_{j}^{(0)}>\right)(\Delta \psi-\mathcal{W} \psi)_{i}\right)_{k}= \\
& =\sum_{k 1} \mathcal{B}_{k, k 1}^{-1} \sum_{i}\left(\delta_{J} \sum_{m} \bar{\psi}_{m, k 1}<J\left(p_{i}, p_{m}\right), p_{j}^{(0)}>\right)(\Delta \psi-\mathcal{W} \psi)_{i, k 1}= \\
& =\sum_{k 1} \mathcal{B}_{k, k 1}^{-1} \sum_{i}\left(\delta_{J} \sum_{m} \bar{\psi}_{m, k 1}<J\left(p_{i}, p_{m}\right), p_{j}^{(0)}>\right) \sum_{k 2} \mathcal{B}_{k 1, k 2} \Theta_{i, k 2}^{*}= \\
& =\sum_{k 2} \sum_{i} \underbrace{\sum_{k 1} \mathcal{B}_{k, k 1}^{-1}\left(\delta_{J} \sum_{m} \bar{\psi}_{m, k 1}<J\left(p_{i}, p_{m}\right), p_{j}^{(0)}>\right) \mathcal{B}_{k 1, k 2}}_{J_{(k, k 2),(j, i)}^{(1)}(\bar{\psi})} \Theta_{i, k 2}^{*} .
\end{aligned}
$$

The second term of the right hand side of (40) we rewrite using (48), (43) as

$$
\begin{aligned}
& -\left(\mathcal{B}^{-1} \sum_{i}\left(\delta_{J} \sum_{m}(\Delta \bar{\psi}+\beta y-\mathcal{W} \bar{\psi})_{m}<J\left(p_{i}, p_{m}\right), p_{j}^{(0)}>\right) \psi_{i}\right)_{k}= \\
& -\sum_{k 1} \mathcal{B}_{k, k 1}^{-1} \sum_{i}\left(\delta_{J} \sum_{m}(\Delta \bar{\psi}+\beta y-\mathcal{W} \bar{\psi})_{m, k 1}<J\left(p_{i}, p_{m}\right), p_{j}^{(0)}>\right) \psi_{i, k 1}= \\
& -\sum_{k 1} \mathcal{B}_{k, k 1}^{-1} \sum_{i}\left(\delta_{J} \sum_{m}(\Delta \bar{\psi}+\beta y-\mathcal{W} \bar{\psi})_{m, k 1}<J\left(p_{i}, p_{m}\right), p_{j}^{(0)}>\right) \sum_{k 2} \mathcal{B}_{k 1, k 2} \psi_{i, k 2}^{*}= \\
& -\sum_{k 1} \mathcal{B}_{k, k 1}^{-1} \sum_{i}\left(\delta_{J} \sum_{m}(\Delta \bar{\psi}+\beta y-\mathcal{W} \bar{\psi})_{m, k 1}<J\left(p_{i}, p_{m}\right), p_{j}^{(0)}>\right) \sum_{k 2} \mathcal{B}_{k 1, k 2} \sum_{l} \mathcal{H}_{k 2,(i, l)} \Theta_{k 2, l}^{*}= \\
& -\sum_{k 2} \sum_{l} \underbrace{\sum_{k 1} \mathcal{B}_{k, k 1}^{-1} \sum_{i}\left(\delta_{J} \sum_{m}(\Delta \bar{\psi}+\beta y-\mathcal{W} \bar{\psi})_{m, k 1}<J\left(p_{i}, p_{m}\right), p_{j}^{(0)}>\right) \mathcal{B}_{k 1, k 2} \mathcal{H}_{k 2,(i, l)} \Theta_{k 2, l}^{*}(53)}_{J_{(k, k 2),(j, l)}^{(2)}(\bar{\psi})}
\end{aligned}
$$

The following two terms of (40) describing the linear dissipation do not depend on the basic solution. The lateral friction term we write using (48) :

$$
\begin{aligned}
& \delta_{A}\left(-\mathcal{C}^{(1 / 2)} \Theta_{k}^{*}+\Lambda_{k} \mathcal{M}^{(1 / 2)} \Theta_{k}^{*}+\Lambda_{k}^{2} \mathcal{M}^{(1 / 2)} \psi_{k}^{*}\right)= \\
= & \delta_{A} \underbrace{\left(-\mathcal{C}^{(1 / 2)}+\Lambda_{k} \mathcal{M}^{(1 / 2)}+\Lambda_{k}^{2} \mathcal{M}^{(1 / 2)} \mathcal{H}_{k}\right)}_{\mathcal{A}_{k}} \Theta_{k}^{*}
\end{aligned}
$$

and the bottom drag

$$
-\delta_{\varepsilon} \mathcal{V}\left(\mathcal{M}^{(1 / 2)} \Theta^{*}+\Lambda \mathcal{M}^{(1 / 2)} \psi^{*}\right)_{k}=-\delta_{\varepsilon} \sum_{k 1} \underbrace{\mathcal{V}_{k, k 1}\left(\mathcal{M}^{(1 / 2)}+\Lambda_{k 1} \mathcal{M}^{(1 / 2)} \mathcal{H}_{k 1}\right)}_{\mathcal{D}_{k, k 1}} \Theta_{k 1}^{*}
$$

Finally we obtain the matricial formulation of $(40)$ :

$\operatorname{RR} \mathrm{n}^{\circ} 3074$

$$
\mathcal{M}^{(0)} \frac{\partial \Theta_{k}^{*}(0)}{\partial t}=\sum_{k 1} \hat{J}_{(k, k 1)}(\bar{\psi}) \Theta_{k 1}^{*}-\sum_{k 1} \mathcal{D}_{k, k 1} \Theta_{k 1}^{*}+\mathcal{A}_{k} \Theta_{k}^{*}
$$


where

$$
\hat{J}_{(k, k 1)}(\bar{\psi})=\hat{J}_{(k, k 1)}^{(1)}(\bar{\psi})-\hat{J}_{(k, k 1)}^{(2)}(\bar{\psi})
$$

If we represent the discretised functions in all $K$ layers as vectors- columns of length $K \times N$

$$
\Theta=\left(\Theta_{1,1}, \ldots, \Theta_{1, N}, \ldots, \Theta_{K, 1}, \ldots, \Theta_{K, N}\right)^{T}
$$

we obtain that operator of the equation (56) can be presented as a block-matrix composed of $K \times K$ blocks of $N^{(0)} \times N$ size each.

$$
\mathcal{G}=\left\{\begin{array}{cccc}
\hat{J}_{(1,1)}-\mathcal{D}_{1,1}+\mathcal{A}_{1} & \hat{J}_{(1,2)}-\mathcal{D}_{1,2} & \ldots & \hat{J}_{(1, K)}-\mathcal{D}_{1, K} \\
\hat{J}_{(2,1)}-\mathcal{D}_{2,1} & \hat{J}_{(2,2)}-\mathcal{D}_{2,2}+\mathcal{A}_{2} & \ldots & \hat{J}_{(2, K)}-\mathcal{D}_{2, K} \\
\hat{J}_{(K, 1)}-\mathcal{D}_{K, 1} & \hat{J}_{(K, 2)}-\mathcal{D}_{K, 2} & \ldots & \hat{J}_{(K, K)}-\mathcal{D}_{K, K}+\mathcal{A}_{K}
\end{array}\right\}
$$

We note here that the matrix $\mathcal{G}$ is composed of rectangular blocks. This rectangularity reflects that we look for the function $\Theta_{k}^{*(0)}$ which is composed of $N^{(0)}$ elements being equal to zero on the boundary while the function $\Theta_{k}^{*}$ is composed of $N$ elements.

So the equation (56) will have a simple linear form:

$$
\mathcal{M}^{(0)} \frac{\partial \Theta^{*(0)}}{\partial t}=\mathcal{G}(\bar{\psi}(t)) \Theta^{*}
$$

where $\left(\mathcal{G} \Theta^{*}\right)_{i, k}=\sum_{k 1}^{K} \sum_{j}^{N} \mathcal{G}_{(i, j),(k, k 1)} \Theta_{j, k 1}, \quad i=1, \ldots, N^{(0)}$.

\subsection{Evolution of the boundary constants.}

To obtain the evolution of the function $\Theta^{*(0)}$ we apply some numerical scheme of integration in time. The choice of this scheme is based on the purpose of calculations. Since we are interested in the evolution of the error basis in the phase space, the cost of calculations will be relatively high: we have to perform integration of each vector of the basis. While the simple integration of the model equation requires at most $O(K \times N)^{2}$ operations per time step, the predictability calculations require $O(K \times N)^{3}$ operations on each time step. So we need to make the time step as large as possible and it would be better if we are capable to choose it from the approximation condition only rather than from the condition of the stability of the scheme. Another aspect of our calculations concerns the conservation properties. We need to calculate the evolution of a norm of a perturbation, so our scheme must possess a conservative qualities. In other words no numerical dissipation is allowed. Basing on these two principles we choose the scheme of Crank-Nicholson which is absolutely stable and conservative. This choice is not very popular for ordinary integration of differential equations so far this scheme is implicit, therefore it requires the resolution of a linear system of equations on each time step. This procedure sharply increases the number of operations and calculation time. However for our task the augmentation of time with respect to some explicit scheme is not so great due to high cost of a step even for a simple explicit scheme. Below we perform the numerical comparison of the accuracy and the cost of different schemes applied to our calculations and show that the scheme of Crank-Nicholson satisfies our requirements in the best way. 
From now and further we drop the stars designating that functions are considered in the eigenbasis. We apply the Crank-Nicholson scheme for integration of the equation (58).

$$
\mathcal{M}^{(0)} \frac{\Theta_{k}^{(0), n+1}-\Theta_{k}^{(0), n}}{\tau}=\sum_{k 1}^{K} \mathcal{G}_{(k, k 1)}\left(\bar{\psi}^{n+1 / 2}\right) \frac{\Theta_{k 1}^{n+1}+\Theta_{k 1}^{n}}{2}
$$

Taking into account (27), we obtain

$$
\begin{array}{r}
\sum_{k 1}^{K} \mathcal{G}_{(k, k 1)}\left(\bar{\psi}^{n+1 / 2}\right) \Theta_{k 1}^{n+1}=\sum_{k 1}^{K} \sum_{j}^{N} \mathcal{G}_{(i, j),(k, k 1)}\left(\bar{\psi}^{n+1 / 2}\right)\left(\Theta_{j, k 1}^{(0), n+1}-\Lambda_{k 1} C_{k 1} \Theta_{j, k 1}^{(1)}\right)= \\
\sum_{k 1}^{K} \sum_{j}^{N^{(0)}} \mathcal{G}_{(i, j),(k, k 1)}^{(0)}\left(\bar{\psi}^{n+1 / 2}\right) \Theta_{j, k 1}^{(0), n+1}-\sum_{k 1}^{K} \sum_{j}^{N} \mathcal{G}_{(i, j),(k, k 1)}\left(\bar{\psi}^{n+1 / 2}\right) \Lambda_{k 1} C_{k 1} \Theta_{j, k 1}^{(1)}
\end{array}
$$

Thanks to the fact that $\Theta^{(0)}$ is equal to zero on the boundary we can substitute the operator $\mathcal{G}^{(0)}$ for $\mathcal{G}$. Elements of the operator $\mathcal{G}^{(0)}$ are equal to corresponding elements of $\mathcal{G}$ for all $j: 1 \leq j \leq N^{(0)}$ but contrary to $\mathcal{G}$ the operator $\mathcal{G}^{(0)}$ is composed of squared blocks of $N^{(0)} \times N^{(0)}$ size.

Thanks to (44) we can write

$$
\mathcal{M}^{(1 / 2)} \Theta_{k}=\mathcal{M}^{(1 / 2)}\left(\Theta_{k}{ }^{(0)}-\Lambda_{k} C_{k} \Theta_{k}^{(1)}\right)=\mathcal{M}^{(1 / 2)} \Theta_{k}{ }^{(0)}=\mathcal{M}^{(0)} \Theta_{k}{ }^{(0)} .
$$

Thus we have

$$
\begin{aligned}
\sum_{k 1}^{K}\left(\mathcal{M}^{(0)} \delta_{k, k 1}-\frac{\tau}{2} \mathcal{G}_{(k, k 1)}^{(0), n+1 / 2}\right) \Theta_{k 1}^{(0), n+1}+ & \frac{\tau}{2} \sum_{k 1}^{K} \mathcal{G}_{(k, k 1)}^{n+1 / 2} \Lambda_{k 1} \Theta_{k 1}^{(1)} C_{k 1}^{n+1}= \\
& =\sum_{k 1}^{K}\left(\mathcal{M}^{(1 / 2)} \delta_{k, k 1}+\frac{\tau}{2} \mathcal{G}_{(k, k 1)}^{n+1 / 2}\right) \Theta_{k 1}^{n} .
\end{aligned}
$$

We can obtain the value of the boundary constant at $n+1$ time step from (28), (41). We have

$$
C_{k}^{n+1}=\frac{\int_{\Omega} \psi_{k}^{t=0} d x d y-\int_{\Omega} \psi_{k}^{(0), n+1} d x d y}{\int_{\Omega} \psi_{k}^{(1)} d x d y}=\frac{D_{k}^{t=0}-D_{k, n+1}^{(0)}}{D_{k}^{(1)}}
$$

and

$$
\psi_{k}^{(0)}=-\left(\mathcal{C}^{(0)} \psi_{k}^{(0)}+\Lambda_{k} \mathcal{M}^{(0)}\right)^{-1} \mathcal{M}^{(0)} \Theta_{k}^{(0)} \equiv \mathcal{H}_{k}^{(0)} \Theta_{k}^{(0)}
$$

Therefore

$$
D_{k, n+1}^{(0)}=\int_{\Omega} \psi_{k}^{(0), n+1} d x d y=\sum_{i}^{N^{(0)}} \alpha_{i} \psi_{i, k}^{(0), n+1}=\sum_{i}^{N^{(0)}} \alpha_{i} \sum_{j}^{N^{(0)}} \mathcal{H}_{i, j}^{k,(0)} \Theta_{j, k}^{(0), n+1}=\sum_{j}^{N^{(0)}} \xi_{j, k}^{(0)} \Theta_{j, k}^{(0), n+1},
$$

where $\alpha_{i}$ are weights used in the integration, and

$\operatorname{RR} n^{\circ} 3074$

$$
\xi_{j, k}^{(0)}=\sum_{i}^{N^{(0)}} \alpha_{i} \mathcal{H}_{i, j}^{k,(0)}
$$


So far the boundary constants $C_{k}(t)$ are always chosen to satisfy the condition (13) of conservation of the integral $\int \psi_{k}(t) d x d y=\int \psi_{k}^{t=0} d x d y \quad \forall t$, we can substitute $\int \psi_{k}^{n} d x d y$ for $\int \psi_{k}^{t=0} d x d y$ in (62). This allows us to use $D_{k}^{n}$ instead of $D_{k}^{t=0}$ :

$$
D_{k}^{t=0}=D_{k}^{n}=\int_{\Omega} \psi_{k}^{n} d x d y=\sum_{i}^{N} \alpha_{i} \psi_{i, k}^{n}=\sum_{i}^{N} \alpha_{i} \sum_{j}^{N} \mathcal{H}_{i, j}^{k} \Theta_{j, k}^{n}=\sum_{j}^{N} \xi_{j, k} \Theta_{j, k}^{n},
$$

with operator $\mathcal{H}$ taken from (48), and $\xi_{j, k}=\sum_{i}^{N} \alpha_{i} \mathcal{H}_{i, j}^{k}$.

Using these formulae we can write the second term of $(61)$

$$
\begin{aligned}
& \frac{\tau}{2} \sum_{k 1}^{K} \mathcal{G}_{(k, k 1)}^{n+1 / 2} \Lambda_{k 1} \Theta_{k 1}^{(1)} C_{k 1}^{n+1}=\frac{\tau}{2} \sum_{k 1}^{K} \sum_{j}^{N} \mathcal{G}_{(k, k 1),(i, j)}^{n+1 / 2} \frac{\Lambda_{k 1}}{D_{k 1}^{(1)}} \Theta_{j, k 1}^{(1)}\left(\sum_{l}^{N} \xi_{l, k 1} \Theta_{l, k 1}^{n}-\sum_{l}^{N^{(0)}} \xi_{l, k 1}^{(0)} \Theta_{l, k 1}^{(0), n+1}\right)= \\
& =\frac{\tau}{2} \sum_{k 1}^{K} \sum_{l}^{N} \underbrace{\xi_{l, k} \sum_{j}^{N} \mathcal{G}_{(k, k 1),(i, j)}^{n+1 / 2} \frac{\Lambda_{k 1}}{D_{k 1}^{(1)}} \Theta_{j, k 1}^{(1)}}_{\chi_{(k, k 1),(i, l)}^{n}} \Theta_{l, k 1}^{n}-\frac{\tau}{2} \sum_{k 1}^{K} \sum_{l}^{N^{(0)}} \underbrace{\xi_{l, k} \sum_{j}^{N} \mathcal{G}_{(k, k 1),(i, j)}^{n+1 / 2} \frac{\Lambda_{k 1}}{D_{k 1}^{(1)}} \Theta_{j, k 1}^{(1)}}_{\chi_{(k, k 1),(i, l)}^{(0),}} \Theta_{l, k 1}^{(0), n+1}= \\
& =\frac{\tau}{2} \sum_{k 1}^{K} \chi_{k, k 1}^{n} \Theta_{k 1}^{n}-\frac{\tau}{2} \sum_{k 1}^{K} \chi_{k, k 1}^{(0), n} \Theta_{k 1}^{(0), n+1} .
\end{aligned}
$$

So we rewrite the equation (61)

$$
\begin{aligned}
\sum_{k 1}^{K}(\underbrace{\mathcal{M}^{(0)} \delta_{k, k 1}-\frac{\tau}{2} \mathcal{G}_{k, k 1}^{(0), n+1 / 2}-\frac{\tau}{2} \chi_{k, k 1}^{(0), n}}_{T_{(0)}^{n}}) \Theta_{k 1}^{(0), n+1}= \\
=\sum_{k 1}^{K}\left(\mathcal{M}^{1 / 2} \delta_{k, k 1}+\frac{\tau}{2} \mathcal{G}_{k, k 1}^{n+1 / 2}-\frac{\tau}{2} \chi_{k, k 1}^{n}\right) \Theta_{k 1}^{n}
\end{aligned}
$$

or simply

$$
\begin{aligned}
\Theta_{k}^{(0), n+1}= & T_{(0), n}^{-1}\left(\mathcal{M}^{1 / 2}+\frac{\tau}{2} \mathcal{G}^{n+1 / 2}-\frac{\tau}{2} \chi_{(k, k 1)}^{n}\right) \Theta^{n} \equiv \sum_{k 1}^{K} \mathcal{T}_{k, k 1}^{n} \Theta_{k 1}^{n} \\
& \text { with } \mathcal{T}^{n}=T_{(0)}^{-1}\left(\mathcal{M}^{1 / 2}+\frac{\tau}{2} \mathcal{G}^{n+1 / 2}-\frac{\tau}{2} \chi_{(k, k 1)}^{n}\right) .
\end{aligned}
$$

We can say that the operator $T$ is inversible when $\tau$ is sufficiently small.

However we are interested in the evolution of function with boundary conditions (17) rather than with Dirichlet ones. So we look for the value $\Theta_{k}^{n+1}$ at $n+1$ time step. From $(62),(64),(65)$ and (68) we have

$$
\begin{aligned}
& \Theta_{i, k}^{n+1}=\Theta_{i, k}^{(0), n+1}-\Lambda_{k} C_{k}^{n+1} \Theta_{i, k}^{(1)}= \\
= & \sum_{k 1}^{K} \sum_{l}^{N} \mathcal{T}_{(k, k 1),(i, l)}^{n} \Theta_{k 1, l}^{n}-\frac{\Lambda_{k}}{D_{k}^{(1)}}\left(\sum_{j}^{N} \xi_{j, k} \Theta_{j, k}^{n}-\sum_{j}^{N^{(0)}} \xi_{j, k}^{(0)} \Theta_{j, k}^{(0), n+1}\right) \Theta_{i, k}^{(1)}=
\end{aligned}
$$




$$
\begin{aligned}
& =\sum_{k 1}^{K} \sum_{l}^{N} \mathcal{T}_{(k, k 1),(i, l)}^{n} \Theta_{k 1, l}^{n}+\frac{\Lambda_{k}}{D_{k}^{(1)}} \sum_{j}^{N^{(0)}} \xi_{j, k}^{(0)} \sum_{k 1}^{K} \sum_{l}^{N} \mathcal{T}_{(k, k 1),(j, l)}^{n} \Theta_{k 1, l}^{n} \Theta_{i, k}^{(1)}-\frac{\Lambda_{k}}{D_{k}^{(1)}} \sum_{j}^{N} \xi_{j, k} \Theta_{j, k}^{n} \Theta_{i, k}^{(1)}= \\
& =\sum_{k 1}^{K} \sum_{l}^{N} \mathcal{T}_{(k, k 1),(i, l)}^{n} \Theta_{k 1, l}^{n}+\sum_{k 1}^{K} \sum_{l}^{N} \underbrace{\frac{\Lambda_{k}}{D_{k}^{(1)}} \sum_{j}^{N^{(0)}} \xi_{j, k} \mathcal{T}_{(k, k 1),(j, l)}^{n} \Theta_{i, k}^{(1)}}_{\zeta_{i, l}^{k, k 1}} \Theta_{k 1, l}^{n}-\sum_{j}^{N} \underbrace{\frac{\Lambda_{k}}{D_{k}^{(1)}} \xi_{j, k} \Theta_{i, k}^{(1)}}_{\eta_{i, j}^{k}} \Theta_{j, k}^{n}= \\
& =\sum_{k 1}^{K} \sum_{j}^{N}\left(\mathcal{T}_{(k, k 1),(i, j)}^{n}+\zeta_{i, j}^{k, k 1}-\eta_{i, j}^{k} \delta_{k, k 1}\right) \Theta_{k 1, j}^{n} .
\end{aligned}
$$

In matricial formulation we obtain

$$
\Theta^{n+1}=\left(\mathcal{T}^{n}+\zeta^{n}-\eta\right) \Theta^{n}
$$

where

$$
\begin{aligned}
\mathcal{T}_{k, k 1}^{n} & =\left(\mathcal{M}^{(0)} \delta_{k, k 1}-\frac{\tau}{2} \mathcal{G}_{k, k 1}^{(0), n+1 / 2}-\frac{\tau}{2} \chi_{(k, k 1)}^{(0), n}\right)^{-1}\left(\mathcal{M}^{(1 / 2)}+\frac{\tau}{2} \mathcal{G}^{n+1 / 2}-\frac{\tau}{2} \chi_{(k, k 1)}^{n}\right), \\
\zeta_{(i, j),(k, k 1)}^{n} & =\frac{\Lambda_{k}}{D_{k}^{(1)}} \sum_{l}^{N^{(0)}} \xi_{l, k} \mathcal{T}_{(k, k 1),(l, j)}^{n} \Theta_{i, k}^{(1)}, \quad i=1, \ldots, N, \quad j=1, \ldots, N \\
\eta_{(i, j),(k)} & =\frac{\Lambda_{k}}{D_{k}^{(1)}} \xi_{j, k} \Theta_{i, k}^{(1)} .
\end{aligned}
$$

Let us discuss the sense of operators in the equation (70). First, the operator $\mathcal{T}$ is responsible for the evolution of the potential vorticity $\Theta$ without taking into account boundary conditions. This operator is $K \times K$ block matrix composed of $N^{(0)} \times N$ rectangular blocks. The rectangularity of blocks shows that no calculation of boundary points is performed by this matrix. Each block $k, k 1$ of $\zeta$ is a squared matrix of size $N \times N$. The sum of matrices $\mathcal{T}$ and $\zeta$ produces a projector of an $N$-dimensional space to its subspace defined by the condition

$$
\sum_{l}^{N} \xi_{l, k} \Theta_{l, k}=0, \forall k=2, \ldots, K
$$

This can be easily seen from the following. Let $\theta^{\prime}=(\mathcal{T}+\zeta) \theta$. Then

$$
\begin{array}{r}
\sum_{l}^{N} \xi_{l, k} \theta_{l, k}^{\prime}=\sum_{l}^{N} \xi_{l, k} \sum_{k 1}^{K} \sum_{j}^{N}\left(\mathcal{T}_{(k, k 1),(l, j)}+\zeta_{(k, k 1),(l, j)}\right) \theta_{j, k 1}= \\
=\sum_{k 1}^{K} \sum_{j}^{N} \sum_{l}^{N} \xi_{l, k} \mathcal{T}_{(k, k 1),(l, j)} \theta_{j, k 1}+\sum_{k 1}^{K} \sum_{j}^{N} \sum_{l}^{N} \xi_{l, k} \frac{\Lambda_{k}}{D_{k}^{(1)}} \sum_{m}^{N^{(0)}} \xi_{m, k} \mathcal{T}_{(k, k 1),(m, j)} \Theta_{l, k}^{(1)} \theta_{j, k 1}= \\
=\sum_{k 1}^{K} \sum_{j}^{N} \sum_{l}^{N} \xi_{l, k} \mathcal{T}_{(k, k 1),(l, j)} \theta_{j, k 1}+\sum_{k 1}^{K} \sum_{j}^{N} \sum_{m}^{N^{(0)}}\left(\frac{\Lambda_{k}}{D_{k}^{(1)}} \sum_{l}^{N} \xi_{l, k} \Theta_{l, k}^{(1)}\right) \xi_{m, k} \mathcal{T}_{(k, k 1),(m, j)} \theta_{j, k 1}=0
\end{array}
$$

thanks to

$\operatorname{RR~} \mathrm{n}^{\circ} 3074 \Lambda_{k}\left(\sum_{l}^{N} \xi_{l, k} \Theta_{l, k}^{(1)}\right)=\Lambda_{k} \frac{\sum_{l}^{N} \sum_{i}^{N} \alpha_{i} \mathcal{H}_{i, l}^{k} \Theta_{l, k}^{(1)}}{\sum_{i}^{N} \alpha_{i} \psi_{i, k}^{(1)}}=\Lambda_{k} \frac{-\frac{1}{\Lambda_{k}} \sum_{i}^{N} \alpha_{i} \psi_{i, k}^{(1)}}{\sum_{i}^{N} \alpha_{i} \psi_{i, k}^{(1)}}=-1$ 
The addition of the term $-\eta \Theta^{n}$ in the equation (70) provides that the solution at the $n+1$ time step satisfies the condition $\int_{\Omega} \psi_{k}^{n+1} d \Omega=\int_{\Omega} \psi_{k}^{n} d \Omega$ and, consequently, $\int_{\Omega} \psi_{k}^{n+1} d \Omega=$ $\int_{\Omega} \psi_{k}^{t=0} d \Omega$ for $k=2,3, \ldots, K$. The only non zero eigenvalue of the operator $\eta$ corresponds to the eigenvector $\Theta_{k}^{(1)}$. Hence addition of $\eta \Theta^{n}$ results simply in addition of $\Theta_{k}^{(1)}$, normalized so to satisfy $\int_{\Omega} \psi_{k}^{n+1} d \Omega=\int_{\Omega} \psi_{k}^{n} d \Omega$.

\section{Lyapunov exponents of quasi-geostrophic circulation.}

\subsection{Predictability characteristics on different time scales.}

We have obtained the equation governing the evolution of a small perturbation of initial conditions of the quasi-geostrophic model. This equation is derived in the linear approach, therefore we have to work with infinitesimal perturbations only. This rather strong restriction used in this paper limits the scope of the predictability study of a physical system. First, no physical perturbation of initial conditions is infinitesimal because of finite accuracy of data measurements. And second, the perturbation must remain infinitesimal during all the integration time, even in the case when $T$ is large. The linear approach assumes the initial error can grow infinitely, while a finite error growth is limited by the size of the attractor.

Despite these two restrictions this study could provide us with an important information about the system. Considering the evolution of a finite error, we can frequently assume that initial error is sufficiently small to be considered as infinitesimal. If we study the error evolution during short time interval we can suppose that the error remains small and hence withing frames of the linear approach.

Furthermore, consideration of infinitesimal perturbations allows us to obtain global predictability characteristics of a system like its Lyapunov exponents which relates to infinite time behaviour of an infinitesimal error. Performing long time integration of a system we can estimate its attractor dimension and Kolmogorov's entropy which characterize the average rate of loosing information by the system.

Let us consider the evolution of a small perturbation of initial conditions of quasi-geostrophic model given by equation (70). If after one time step the initial error will be equal to

$$
\Theta^{n+1}=\left(\mathcal{T}^{n}+\zeta^{n}-\eta\right) \Theta^{n} \equiv B\left(\bar{\psi}^{n}\right) \Theta^{n}
$$

then after $L$ time steps we obtain

$$
\begin{aligned}
\Theta^{n+L} & =B\left(\bar{\psi}^{n+L-1}\right) \Theta^{n+L-1}=B\left(\bar{\psi}^{n+L-1}\right) B\left(\bar{\psi}^{n+L-2}\right) \Theta^{n+L-2}= \\
& =\prod_{i=0}^{L-1} B\left(\bar{\psi}^{n+i}\right) \Theta^{n} \equiv B^{(L)}\left(\bar{\psi}^{n}\right) \Theta^{n} .
\end{aligned}
$$

It has to be noted here, that the index $(L)$ is not the power of the operator. This index denotes the number of multiplications of different operators $B\left(\bar{\psi}^{n+i}\right)$. This index corresponds to the length of the basic trajectory $\bar{\psi}$ or to the time $L \tau$ during which we let the initial error to grow. Further we shall address this time as the composition length.

To estimate the evolution of its norm we define the scalar product in the finite dimensional space of discretised functions. We shall use the finite dimensional approximation of the scalar 
product in $L_{2}$ functional space:

$$
<\theta, \phi>=\int_{\Omega} \theta \phi d x d y=\sum_{i}^{N} \sum_{j}^{N} \theta_{i} \phi_{j} \int_{\Omega} p_{i} p_{j} d x d y=\sum_{j}^{N} \phi_{j}(\mathcal{M} \theta)_{j}=(\mathcal{M} \theta, \phi) .
$$

The norm of a function defined by this scalar product is

$$
\|\theta\|=<\theta, \theta>^{1 / 2}=(\mathcal{M} \theta, \theta)^{1 / 2} .
$$

Since the mass matrix $\mathcal{M}$ is positive definite, the scalar product $(\mathcal{M} \theta, \theta)$ is positive for all $\theta$. We can obtain the norm of the perturbation after $L$ time steps from (73):

$$
\left\|\Theta^{t=L \tau}\right\|^{2}=\left(\mathcal{M} \Theta^{t=L \tau}, \Theta^{t=L \tau}\right)=\left(\mathcal{M} B^{(L)} \Theta^{t=0}, B^{(L)} \Theta^{t=0}\right)=\left(B^{(L)^{t}} \mathcal{M} B^{(L)} \Theta^{t=0}, \Theta^{t=0}\right)
$$

where $B^{(L)^{t}}$ is the transposed operator.

To study the predictability of the quasi-geostrophic model we should choose some measure of the predictability. One of well known characteristics of predictability of nonlinear dynamical systems is the Lyapunov exponents. They are introduced to describe the growth or decay of an infinitesimal elementary m-dimensional volume around the point $\bar{\psi}^{t=0}(x, y)$ in the limit of large T.

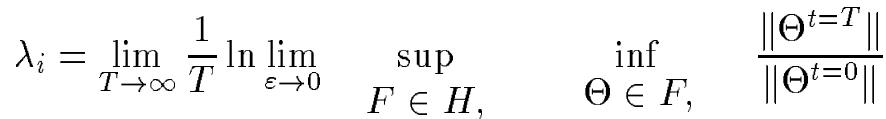

$$
\begin{aligned}
& \operatorname{dim} F=i \quad\left\|\Theta^{t=0}\right\|<\varepsilon
\end{aligned}
$$

For our system the evolution of an error is expressed by (73) in discrete time where the time interval is equal to the product of number of steps and the time step value $T=L \tau$. The evolution of the norm of error is given by (75), so we can calculate Lyapunov exponents as

$$
\lambda_{i}=\lim _{L \rightarrow \infty} \frac{1}{2 L \tau} \ln \lim _{\varepsilon \rightarrow 0} \sup _{\substack{F \in H, \operatorname{dim} F=i}} \inf _{\Theta \in F,} \frac{\left(B^{(L)^{t}} \mathcal{M} B^{(L)} \Theta^{t=0}, \Theta^{t=0}\right)}{\left(\mathcal{M} \Theta^{t=0}, \Theta^{t=0}\right)}
$$

The theorem of Courant-Fisher gives us that the relation of scalar products in the expression (77)

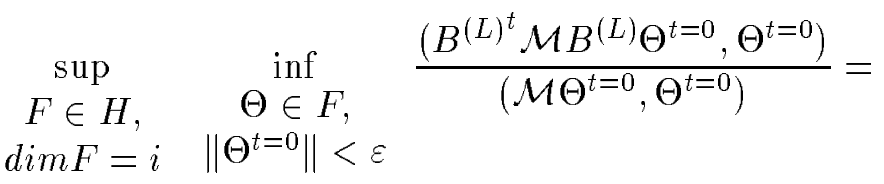

$$
\begin{aligned}
& \begin{array}{c}
\sup _{F \in H,} \inf _{\Theta \in F,} \frac{\left(B^{\left.(L)^{t} \mathcal{M} B^{(L)} \mathcal{M}^{-1} \mathcal{M} \Theta^{t=0}, \Theta^{t=0}\right)}\right.}{\left(\mathcal{M} \Theta^{t=0}, \Theta^{t=0}\right)} \\
\operatorname{dim} F=i \quad\left\|\Theta^{t=0}\right\|<\varepsilon
\end{array}
\end{aligned}
$$

is equal to the $\mathrm{i}$-th eigenvalue of the matrix $B^{(L)^{t}} \mathcal{M} B^{(L)} \mathcal{M}^{-1}$. Therefore we can calculate the Lyapunov exponents $\lambda_{i}$ as

$\operatorname{RR} \mathrm{n}^{\circ} 3074$

$$
\lambda_{i}=\lim _{L \rightarrow \infty} \frac{1}{2 L \tau} \ln \mu_{i}\left(B^{(L)^{t}} \mathcal{M} B^{(L)} \mathcal{M}^{-1}\right)
$$


where $\mu_{i}\left(B^{(L)^{t}} \mathcal{M} B^{(L)} \mathcal{M}^{-1}\right)$ are eigenvalues of the problem

$$
B^{(L)^{t}} \mathcal{M} B^{(L)} \mathcal{M}^{-1} \phi_{i}=\mu_{i} \phi_{i}
$$

ordered as $\mu_{1} \geq \mu_{2} \geq \ldots \geq \mu_{N}$.

Multiplicative ergodic theorem proved in [Oseledets,68] reveals the existence of the limit matrix $\lim _{L \rightarrow \infty}\left(B^{(L)^{t}} \mathcal{M} B^{(L)} \mathcal{M}^{-1}\right)^{1 / 2 L \tau}$. More to the point, if the system is ergodic, Lyapunov exponents (79) do not depend on trajectory (i.e. initial data, except the set of zero measure), they characterize the whole attractor of the system. In other words, if the phase space contains the only basin of attraction, the Lyapunov exponents characterize the attractor in which the trajectory lies rather than the trajectory itself. If the phase space of the system is split into several basins of attraction, then $\lambda_{i}$ can be quite different in these basins. However, even in this case their values relate to characteristics of the basin of attraction defined by trajectory. Further we shall suppose that our system is ergodic, or that there is only one basin of attraction in the phase space.

There are some pieces of information we can extract from these exponents. First, if none is positive, the behaviour of the system is not chaotic. In this case the small perturbation to a trajectory remains near to or approaches that trajectory. In accordance with the theorem [Pesin,77] we can calculate the Kolmogorov-Sinay entropy as the sum of all positive Lyapunov exponents:

$$
K=\sum_{\lambda_{i}>0} \lambda_{i}
$$

This entropy gives us the average rate of loosing information by the system, or in other words the average rate of its trajectory discrepancy. This value gives us the quantitative limits of the predictability of the system. This value we have applied to the study of stability of a stationary point of the atmospheric model to obtain the life time of quasi stationary circulation regimes [Dymnikov et al, 90].

The sum of all Lyapunov exponents of any dissipative system is negative. This sum governs the contraction of a volume in the whole phase space and shows us the existence of the strange attractor. If this sum is positive, the system will "blow up" in time. This fact indicates us that probably we apply wrong physics or we have some kind of numerical instability. If this sum is zero, we have a conservative Hamiltonian system and certainly no strange attractor.

Calculated Lyapunov exponents allow us to calculate the attractor dimension of our system. Let $\mathrm{J}$ be the number of Lyapunov exponents which produce a positive sum, but adding $\lambda_{J+1}$ we obtain the sum negative: $\sum_{i=1}^{J} \lambda_{i} \geq 0$, but $\sum_{i=1}^{J+1} \lambda_{i}<0$. Then the value of dimension of the attractor we can find as [Kaplan, Yorke,79]

$$
D=J+\frac{\sum_{i=1}^{J} \lambda_{i}}{\left|\lambda_{J+1}\right|}
$$

However these exponents, which we shall call global Lyapunov exponents, relate to the infinite time scales of a system behaviour. However in practice we are frequently interested in how perturbations grow or decay during finite time, i.e in a finite number of time step $L$. In meteorological or oceanographical application it is much more important to estimate the 
predictability for a certain period of evolution, say 1 month, rather than for thousands years. So in this paper we consider also the generalization of the notion of Lyapunov exponents introduced in [A barbanel et al(1),91] so that we are able to address local growth rate of instabilities across the attractor. To study the growth or decay of perturbations over a finite lag time from any given point on the attractor we calculate local Lyapunov exponents of the composition length $L \tau$ :

$$
\lambda_{i}^{(L)}(\bar{\psi})=\frac{1}{2 L \tau} \ln \mu_{i}\left(B^{(L)^{t}} \mathcal{M} B^{(L)} \mathcal{M}^{-1}\right)
$$

These exponents characterize the growth of a perturbation made at point $\bar{\psi}$ after a finite number $L$ time steps evolution by the dynamics. If local exponents are large then the possibility to predict in this part of the phase space is rather restricted. On the other hand, if they are small or even negative (even when global exponents might be positive), then the predictability in that part of the phase space is enhanced with respect to the average one. Local Lyapunov exponents $\lambda_{i}^{(L)}(\bar{\psi})$ depend on the particular trajectory $\bar{\psi}$ (or its initial data) and on the period $L \tau$ during which the integration is performed.

The sum of positive local Lyapunov exponents we shall call local Kolmogorov entropy:

$$
K^{(L)}(\bar{\psi})=\sum_{\lambda_{i}^{(L)}>0} \lambda_{i}^{(L)}(\bar{\psi}) .
$$

We shall consider also averages of these exponents over attractor. As the approximation of this average we use averaging over large number of trajectories $\bar{\psi}_{m}$ started from different initial points on the attractor. To be sure that initial points belong to the attractor we choose them as different points on the same trajectory obtained by long time integration of model equations.

$$
\bar{\lambda}_{i}^{(L)}=\lim _{M \rightarrow \infty} \frac{1}{M} \sum_{m}^{M} \lambda_{i}^{(L)}\left(\bar{\psi}_{m}\right)
$$

Like global exponents defined by (79), the $\bar{\lambda}_{i}^{(L)}$ do not depend on the trajectory, so they are characteristics of the attractor and not of some specific orbit on it. They show us how much, on the average over the attractor, a perturbation behaves in time $L \tau$.

It has to be noted that average local Lyapunov exponents are not equal to the global ones. Moreover, they can differ a lot for some systems. It is the case, as we shall show, of our system: average local exponents with low $L$ can be 100 times higher than global ones. It means that the growth rate of a perturbation to a system state in short time scale is higher than the growth rate in long scales. So the local predictability is lower than the global one.

In [Dymnikov et al, 93] we have shown that when the time $L \tau$ grows

- the largest average local Lyapunov exponent $\bar{\lambda}_{i}^{(L)}$ does not grow,

- the average local Kolmogorov entropy $\bar{K}^{(L)}(\bar{\psi})$ does not grow,

- the total sum of average local Lyapunov exponents does not depend on $L \tau$, i.e. remains constant.

Along with the consideration of averaged values of local Lyapunov exponents we shall study their variations in the phase space examining their probability distributions $P\left(\lambda_{i}^{(L)}(\bar{\psi})\right)$. These $\operatorname{RR} n^{\circ} 3074$ 
distributions show us the normalized number of events when the value $\lambda_{i}^{(L)}(\bar{\psi})$ appears when we take different part of the trajectory $\bar{\psi}$ on the attractor. They do not show where on the attractor the $\lambda_{i}^{(L)}(\bar{\psi})$ is large or small, but they reveal the frequency of occurence of various values of local exponents. This provide us with the information as about the mean values of local exponents and about their moments. As well as mean values, these moments, being independent on the particular trajectory, characterize the attractor of a system. The second moment, for example, indicates the variations of predictability skill in its different parts. In [Abarbanel et al (2), 91] these distributions have been studied numerically for different simple systems like Lorenz model and Hénon map. Analysis of the form of decreasing of moments of probability distributions when mean values of local exponents converge to global ones as $L \rightarrow \infty$ have been proposed there.

And finally, we shall use another type of Lyapunov exponents: the instantaneous ones ([Dymnikov et al, 92]). We consider them as predictability characteristics at a point on the attractor, i.e. we study the evolution of an error made in initial conditions of a trajectory of infinitesimal length issued at this point. These exponents could be useful in the forecasting of the predictability of a system, especially when we know the initial point of the trajectory only.

$$
\begin{aligned}
\lambda_{i}^{(I)}(\bar{\psi}) & =\lim _{\tau \rightarrow 0} \frac{1}{\tau} \ln \lim _{\varepsilon \rightarrow 0} \sup _{\substack{F \in H, \operatorname{dim} F=i \quad \inf ^{\Theta \in F},}} \frac{\left\|\Theta^{t=0}\right\|<\varepsilon}{\left\|\Theta^{t=0}\right\|}= \\
= & \lim _{\tau \rightarrow 0} \frac{1}{2 \tau} \ln \mu_{i}\left(B^{\left.(1)^{t} \mathcal{M} B^{(1)} \mathcal{M}^{-1}\right)}\right.
\end{aligned}
$$

We have used instantaneous exponents and instantaneous Kolmogorov entropy in [Dymnikov et al, 92] to study the relationships between predictability and organisation of atmospheric structures. A priory estimates of these exponents for the quasi-geostrophic model discussed here have been obtained in [Bernier,95].

\subsection{Computation of Lyapunov Exponents.}

Following the procedure described above we can calculate the predictability characteristics of quasi-geostrophic circulation of the ocean. This procedure is composed of the following stages:

- Creation of linear operator $\mathcal{G}$ for the equation (58),

- Application of some numerical scheme, taking into account the evolution of boundary constants: (70),

- Calculation of the operator $B^{(L)}$ by the formula (73),

- Eigen value problem (80)

Here we should note that numerical solution of eigenvalues problem (80) may not be easy due to the fact that matrix $B^{(L)}$, being a product of $L$ matrices, is very ill conditioned for high $L$. The condition number calculated for the spectral norm of a autoconjugate positive definite matrix is equal to the relation of the largest eigenvalue to the smallest one. So in our case this number is equal to

$$
\operatorname{cond}\left(B^{(L)^{t}} \mathcal{M} B^{(L)} \mathcal{M}^{-1}\right)=\frac{\mu_{1}}{\mu_{N}}=\exp ^{2 L \tau\left(\lambda_{1}-\lambda_{N}\right)}
$$


So the condition number of this matrix depends exponentially on the space resolution scale and the length of the time interval $L \tau$ and can reach rather high values.

This leads to large errors in eigenvalues obtained by standard algorithms. To be capable to calculate local Lyapunov exponents with long $L \tau$ and to study the convergence of local exponents to global ones when $L \tau \rightarrow \infty$ we shall use the improved Eckmann and Ruelle method described in [Abarbanel et al(1), 91].

This method proposes subsequent QR decomposition of product of the $B\left(\bar{\psi}^{n}\right)$ at $n$ time step and the matrix $Q^{(n-1)}$ from $n-1$ time step:

$$
B\left(\bar{\psi}^{n}\right) Q^{n-1}=Q^{n} R^{n}, \quad Q^{0}=I
$$

To calculate the product $B^{(L)^{t}} \mathcal{M} B^{(L)} \mathcal{M}^{-1}$ we have to perform the $\mathrm{QR}$ decomposition (87) $2 L+2$ times, using matrix $\mathcal{M}^{-1}$ as $B$ for the first time, matrices $B\left(\bar{\psi}^{n}\right), n=1, \ldots, L$ for next $L$ times, matrix $\mathcal{M}$ after that, and matrices $B^{t}\left(\bar{\psi}^{n}\right), n=L, \ldots, 1$ finally. So we generate the unitary matrix $Q$ and a set of upper triangular matrices $R^{i}$ as

$$
B^{(L)^{t}} \mathcal{M} B^{(L)} \mathcal{M}^{-1}=Q_{0}^{2 L+2} R_{0}^{2 L+2} R_{0}^{2 L+1} \ldots R_{0}^{1} \equiv M_{0}
$$

We define matrix $M_{1} \equiv R_{0}^{2 L+2} R_{0}^{2 L+1} \ldots R_{0}^{1} Q_{0}^{2 L+2}$ which is composed by the same $R_{0}^{i}$ matrices, but with the matrix $Q_{0}^{2 L+2}$ on the right, and perform $\mathrm{QR}$ decomposition:

$$
M_{1}=Q_{1}^{2 L+2} R_{1}^{2 L+2} R_{1}^{2 L+1} \ldots R_{1}^{1}
$$

Repeating this procedure we obtain a set of matrices $M_{0}, M_{1}, M_{2}, \ldots, M_{l}$. We can note that all matrices $M_{l}$ have the same eigenvalues. According to a theorem of numerical analysis the matrix $Q_{l}^{2 L+2}$ tends to identity matrix when $l$ grows. So when it will be equal to the identity with required accuracy we obtain Lyapunov exponents we are interested in as the sum of logarithms of diagonal elements of $R_{l}$

$$
\lambda_{i}=\frac{1}{2 L \tau} \sum_{m=1}^{2 L+2} \ln R_{l}^{m}(i, i)
$$

This method is rather expensive with respect to the ordinary QR algorithm applied to the product of matrices. It requires a lot of memory space and it is less effective due to no acceleration of process $(88,89)$ applied. However this method allows us to calculate Lyapunov exponents with a high accuracy for a large $L$ when the condition number of the product $B^{(L)^{t}} \mathcal{M} B^{(L)} \mathcal{M}^{-1}$ reachs $10^{1000}$ while using standard algorithm we are limited by the condition number about $10^{12}$ for 8 -bytes computer arithmetics.

To increase the efficiency of this method we divide the time interval $0 \ldots, L \tau$ into the set of subintervals $0 \ldots L_{1} \tau ;\left(L_{1}+1\right) \tau \ldots L_{2} \tau ; \ldots ;\left(\mathrm{E}_{k-1}+1\right) \tau \ldots L_{k} \tau=L \tau$. The value of $k$ is chosen so that the matrices $B^{(k)^{t}} \mathcal{M} B^{(k)} \mathcal{M}^{-1}$ have a condition number about $10^{12}$, i.e. largest possible for computer arithmetics. These matrices within each interval $\left(\mathrm{L}_{i-1}+1\right) \tau \ldots L_{i} \tau, i=1, \ldots, k$ are calculated by the direct multiplications and the Eckmann-Ruelle procedure $(88,89)$ is applied to these products.

\section{Numerical results.}

As it has been mentioned already, to study the predictability of the QG ocean dynamics we choose a finite element discretisation. The feasibility of refining the triangulation in regions $\mathrm{RR} \mathrm{n}^{\circ} 3074$ 
with intense velocity gradients allows us to work with lower number of grid nodes while the model keeps its eddy-resolving quality . The finite element multi-layer quasi-geostrophic model used in this paper has been developed by Ch. Bernier. The comparison of this model with finite-difference one performed in [Le Provost, Bernier, Blayo, 94] revealed its advantages like high accuracy, flexibility of discretisation of complex domains and low computational cost. The description of this model one can find in [Bernier,90].

All contour pictures presented in this paper have been plotted by the Grid Analysis and Display System developed by Brian Doty (Center for Ocean-Land-Atmosphere Interactions, University of Maryland).

The package MODULEF [Bernadou,88] has been used to perform a triangulation of a domain. This package produce quasi-regular triangulation of the domain basing on the prescribed grid nodes on its boundary. Two grids used in this paper are presented in fig.1. The first of them is the result of the triangulation of a square of unit side length. We require the refining of the triangulation near the western boundary and especially in the middle of the domain where velocity gradients are extremely sharps. This triangulation is composed of 92 triangles. The integration points set, being a union of verteces and mi-edges of triangles, counts 211 noeuds. So the resolution of the grid varies between $1 / 40$ of the side length (about $100 \mathrm{~km}$ ) near the western boundary and $1 / 7$ of the side length (about $550 \mathrm{~km}$ ) near the eastern one.

The second grid represents the triangulation of the North Atlantic. The domain is comprised between $78^{0} \mathrm{~W} \ldots 3^{0} \mathrm{~W}$ in longitude and $15^{0} \mathrm{~N} \ldots 65^{0} \mathrm{~N}$ in latitude. The triangulation is refined near the American coast. It counts 195 triangles and 436 integration points. The resolution of this grid is about the same, i.e. about $75 \mathrm{~km}$ near the American coast and about $400 \mathrm{~km}$ near the European one.

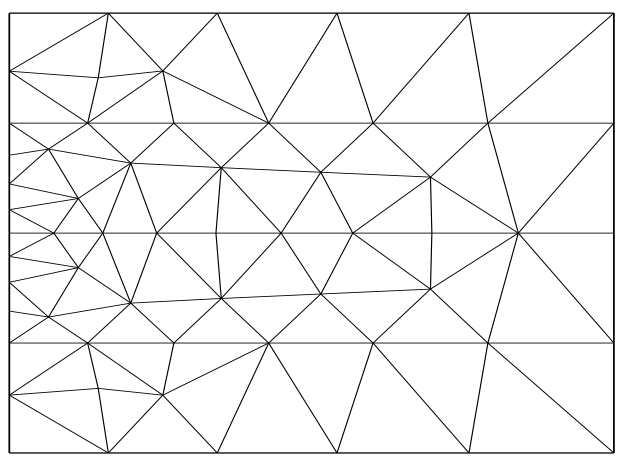

Figure 1A. Triangulation of a unit square.

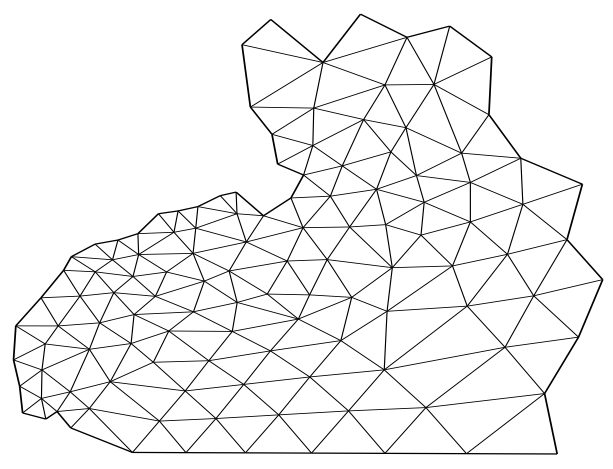

Figure 1B. Triangulation of the North Atlantic.

The numerical study of the predictability of the quasi-geostrophic model is performed basing on 5 experiments. The first of them is the simplest one. We consider the one layer model in a square on the grid presented in fig.1A. Thus the dynamics is supposed to be uniform for any depth of the ocean and the horizontal structure of motions is considered only. 
To study the influence of spatial resolution on the predictability characteristics of this model we perform the second experiment with the same model parameters but with 2 times finer resolution.

The modelisation of vertical structure of the basin is performed in the 3rd experiment where the vertical depth of the ocean is assumed to be composed of two layers. This experiment involves baroclinic dynamics and allows us to see the influence of the baroclinic instability on the predictability characteristics.

More complicated form of the basin is discussed in the 4 th and 5 th experiments. In the fourth experiment we consider the one layer model in the domain which approximates the North Atlantic region in the mid latitudes (fig.1B).

\subsection{Scheme of the integration in time.}

The choice of the scheme of the time integration of the error evolution equation is determined by the lowest integration time of the model. There exists an evident condition that an explicit scheme requires relatively low number of operations per time step, but the value of the time step is restricted by the stability condition of the scheme. On the other hand, the time step of an implicit scheme is limited by the approximation condition only, but the number of operation in this case is relatively higher. For an ordinary integration of a model it is usually clear that some kind of explicit or semi implicit scheme is preferable. However, due to high number of operation per time step in the Lyapunov exponents calculations, this choice is not so evident for the predictability estimates.

To make this choice we compare the accuracy and efficiency of several schemes: the Euler one which is of the first order accuracy, the explicit scheme of the second order and the CrankNicholson implicit scheme. The Euler scheme for the equation (58) can be developed by the same procedure as the equation (70):

$$
\mathcal{M}^{(0)} \frac{\Theta_{k}^{(0), n+1}-\Theta_{k}^{(0), n}}{\tau}=\sum_{k 1}^{K} \mathcal{G}_{(k, k 1)}\left(\bar{\psi}^{n}\right) \Theta_{k 1}^{n}
$$

or in the complete form taking into account the evolution of the boundary constants

$$
\begin{aligned}
& \Theta^{n+1}=\left(\mathcal{T}^{n}+\zeta^{n}-\eta\right) \Theta^{n} \\
& \text { where } \quad \begin{aligned}
\mathcal{T}_{k, k 1}^{n}= & \left(\mathcal{M}^{(0)}\right)^{-1}\left(\mathcal{M}^{(1 / 2)}+\tau \mathcal{G}_{k, k 1}^{n}\right) \\
\zeta_{(i, j),(k, k 1)}^{n}= & \frac{\Lambda_{k}}{D_{k}^{(1)}} \sum_{l}^{N^{(0)}} \xi_{l, k} \mathcal{T}_{(k, k 1),(l, j)}^{n} \Theta_{i, k}^{(1)}, \quad \eta_{(i, j),(k)}=\frac{\Lambda_{k}}{D_{k}^{(1)}} \xi_{j, k} \Theta_{i, k}^{(1)} \\
& i=1, \ldots, N, \quad j=1, \ldots, N .
\end{aligned}
\end{aligned}
$$

The formulation of the Crank-Nicholson scheme for the QG equation is presented in the equation (70). This scheme is stable, so we are less restricted in choice of the timestep.

The important quantity to estimate the scheme quality is the total sum of the Lyapunov exponents. This sum governs the contraction of full $N$-dimensional elementary volume in the phase space.This value depends on the trace of linearized operator ([Temam,88]). So far the trace of Jacobians is 0 , the sum depends on the dissipation only. It can be easily calculated for

$\operatorname{RR} n^{\circ} 3074$ 
our model:

$$
\sum_{i=1}^{K \times N} \lambda_{i}=\frac{A}{L^{2}} \operatorname{Tr}\left(\Delta^{(h)}\right)+\sigma \operatorname{Tr}\left(I^{(h)}\right)
$$

For the grid discussed above the trace of the approximation of the Laplace operator is equal to 619884 . and the trace of the identity operator is equal to the number of integration points 159. For parameters values: $A=2300 \frac{m^{2}}{s}, \sigma=5 \times 10^{-8} s^{-1}$, and the grid this gives $\sum_{i=1}^{K \times N} \lambda_{i}=$ 8.385848 days $^{-1}$.

In the table 1 one can see and compare the accuracy of the explicit Euler and Crank-Nicholson schemes. In spite of the fact that the calculation time by the Crank-Nicholson scheme exceeds the corresponding time required by the Euler scheme, the stability property allows us to use the time step about 20 times larger. If we require, for example, the relative error of the calculation of Lyapunov spectra to be within $5 \%$ interval, we must take the time step about 0.1 day for the Euler scheme. However the use of the Crank-Nicholson scheme allows us to keep the same accuracy with the time step about 2 days. This provides the gain of computation time about 15 times.

Another criterion of the scheme accuracy concerns the calculation of the largest exponents only. The exponents calculated on the same data and the same composition length $L \tau$ must not depend on the number of steps $L$. Thus, for example, we can obtain the composition length $L \tau=25.6$ days either by multiplication of $L=256$ operators with $\tau=0.1$ day, or with $L=1, \tau=25.6$ days. To produce the table 2 we choose time steps and $L$ so to obtain that values in each line correspond to some particular composition length: the last line in each group to 25.6 days, the last by one line to 6.4 days, etc. One can easily see that the Euler scheme produce relative error about $50 \%$ even for $\tau=0.4$ day, while for the Crank-Nicholson scheme the relative error remains always withing $10 \%$ interval.

Table 1. Comparison of time schemes.

\begin{tabular}{|r|r|c|c|c|c|c|c|}
\hline & & \multicolumn{3}{|c|}{ Euler } & \multicolumn{3}{|c|}{ Crank-Nicholson } \\
\hline$\tau$ (day) & $L$ & $\lambda_{\max }$ & $\sum \lambda$ & CPU time & $\lambda_{\max }$ & $\sum \lambda$ & CPU time \\
\hline 0.1 & 1 & 0.88 & -8.11 & 420 & 0.88 & -8.3852 & 580 \\
& 4 & 0.87 & -8.11 & 250 & 0.87 & -8.3852 & 380 \\
& 16 & 0.73 & -8.11 & 210 & 0.73 & -8.3852 & 330 \\
& 64 & 0.34 & -8.11 & 185 & 0.34 & -8.3852 & 300 \\
& 256 & 0.11 & -8.11 & 150 & 0.11 & -8.3852 & 270 \\
\hline 0.4 & 1 & 0.87 & -7.38 & 100 & 0.87 & -8.379 & 130 \\
& 4 & 0.74 & -7.38 & 60 & 0.73 & -8.379 & 90 \\
& 16 & 0.37 & -7.38 & 50 & 0.35 & -8.379 & 85 \\
& 64 & 0.16 & -7.38 & 40 & 0.12 & -8.379 & 80 \\
\hline 1.6 & 1 & 0.78 & -4.72 & 23 & 0.74 & -8.25 & 30 \\
& 4 & 0.41 & -4.72 & 13 & 0.35 & -8.25 & 25 \\
& 16 & 0.31 & -4.72 & 10 & 0.13 & -8.25 & 20 \\
\hline 6.4 & 1 & 0.39 & +0.26 & 5 & 0.34 & -7.45 & 8 \\
& 4 & 0.28 & +0.32 & 3 & 0.13 & -7.45 & 5 \\
\hline 25.6 & 1 & 0.15 & +5.52 & 1 & 0.12 & -3.53 & 1 \\
\hline
\end{tabular}




\subsection{One layer model in a square.}

The simplest version of the quasi-geostrophic model we use in our experiment is the one layer model in a square. It is well known that wind driven barotropic ocean circulation can produce chaotic behaviour under some parameter range (see [Le Provost, Verron,87] for example). The only physical reason which leads the system to chaos is the barotropic instability of the flow. This type of instability has been long discussed for as atmospheric and oceanic dynamics.

We shall consider this experiment as some kind of "reference experiment" to be compared further with experiments with finer resolution grid and with 2 layers model.

In this experiment we take characteristic depth and length of the basin $H$ and $L$ as $5000 \mathrm{~m}$ and $4000 \mathrm{~km}$ respectively. The mean wind field is approximated by a steady zonal wind composed of two gyres antisymmetric pattern:

$$
\mathcal{F}(y)=-\tau_{0} \sin \frac{2 \pi y}{L}
$$

The value of $\tau_{0}$ has been chosen as $5 \times 10^{-13} s^{-2}$, i.e. the wind tension is assumed to be about $\frac{\tau_{0} \rho L H}{2 \pi}=1.6 \frac{\mathrm{N}}{\mathrm{m}^{2}}$. Using the linear Sverdrup balance

$$
\beta \frac{\partial \psi}{\partial x}=\tau_{0}, \text { or } U=\frac{\tau_{0}}{\beta}
$$

one can obtain the characteristic value of velocity of the flow $U=2.5 \mathrm{~cm} / \mathrm{s}$. The coefficient of Ekman dissipation we choose as $\sigma=5 \times 10^{-8} s^{-1}$, which corresponds to the damping timescale $T_{\sigma}=2 \times 10^{7} s \sim 200$ days. The lateral friction coefficient $A$ has been chosen in order to avoid numerical instability which occurs due to the concentration of variability of the model at grid scales. This value has been taken to be $A=2300 \frac{m^{2}}{s}$, that corresponds to the damping time scale $T_{A}=0.64$ day for a wave of $100 \mathrm{~km}$ length. Despite this simplified geometry accompanied by very simple forcing (4), this "academic" case has been intensively investigated over the last 15 years in order to study the role of mesoscale eddies in the ocean circulation.

The QG model in this experiment has been integrated during 20 years from zero state. We suppose that after this period the spin-up phase is over and the solution of the model attains its attractor. After the spin-up the model is integrated during 2048 days with the time step 0.1 day. This part of trajectory we use as $\bar{\psi}(x, y, t)$ in $(70)$ to calculate local Lyapunov exponents of the model. Average streamfunction is shown in fig.2A. 


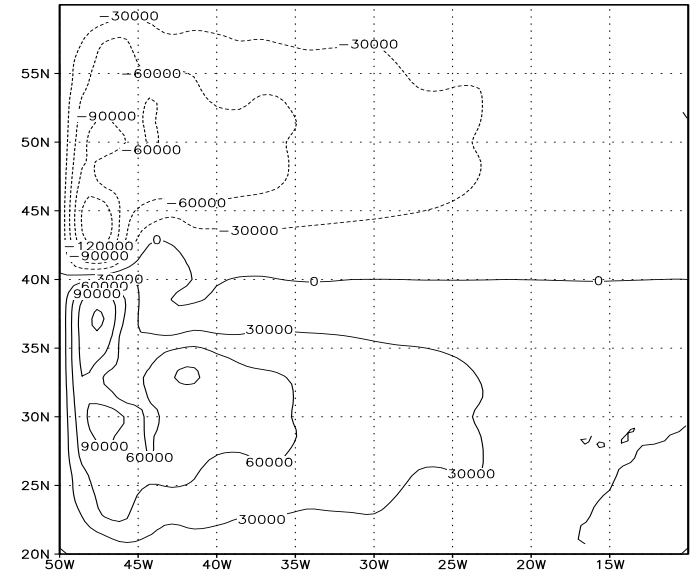

Figure 2A. Time mean streamfunction.

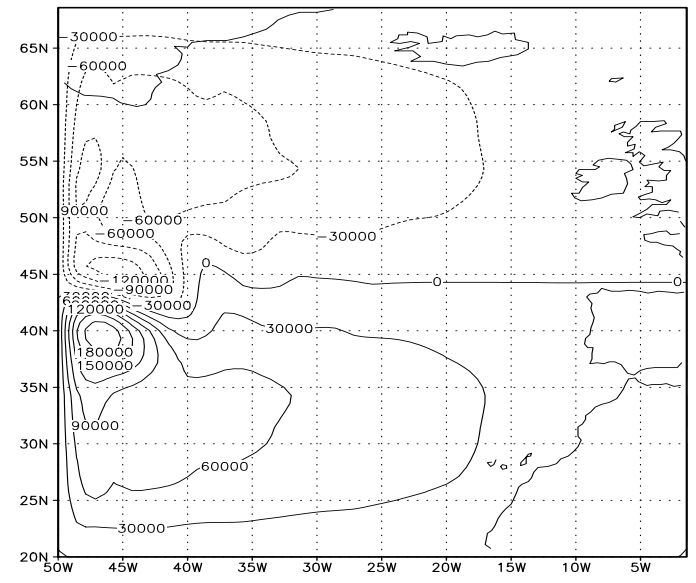

Figure 2B. Time mean streamfunction in the experiment with higher resolution.

To show variations of local exponents we calculate the probability density distribution $P\left(\lambda_{i}^{L}\right)$. Being normalized by unit

$$
\int_{-\infty}^{\infty} P(\lambda) d \lambda=1,
$$

this function can be interpreted as a fraction of time during which the $\mathrm{i}$-th local exponent is equal to the value $\lambda_{i}^{L}$.

In the fig.3A one can see the distributions of 3 largest instantaneous Lyapunov exponents. This distribution is computed by sampling 2048 daily values. In fig.3B the distributions of local Lyapunov exponents calculated by sampling of local exponents calculated for 128 subintervals of 16 days each are presented. Thus this distribution is calculated by 128 samples.

Comparing instantaneous exponents and local exponents calculated for the composition length $L \tau=16$ days (fig.3B) one can remark that along with diminishing of the mean values, the relative dispersion remains almost the same. 


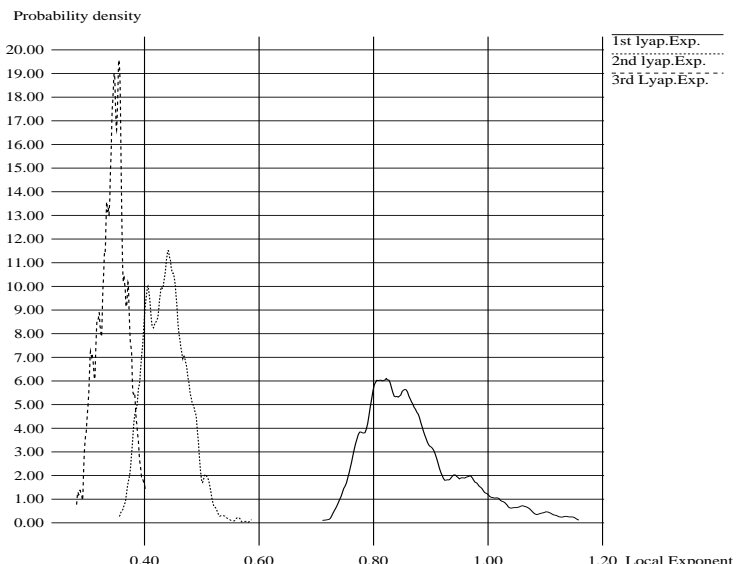

Figure 3A. Distributions of instantaneous Lyapunov Exponents $(L=1)$.

Mean values: $\lambda_{1}=0.87, \lambda_{2}=0.43, \lambda_{3}=0.34$.

Dispersions: $\sigma_{1}=0.085, \sigma_{2}=0.036, \sigma_{3}=0.024$.

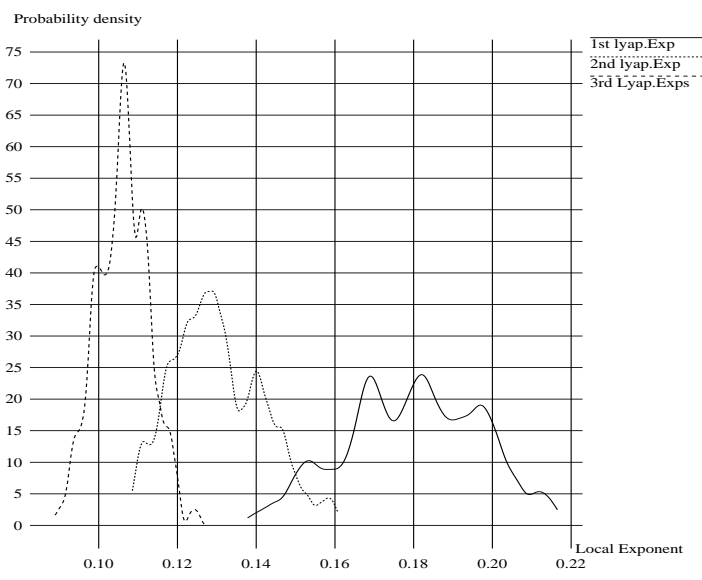

Figure 3B. Distributions of local Lyapunov exponents for $L \tau=16$ days.

Mean values: $\lambda_{1}=0.180, \lambda_{2}=0.130, \lambda_{3}=0.106$.

Dispersions: $\sigma_{1}=0.018, \sigma_{2}=0.012, \sigma_{3}=0.007$.

On fig.3A one can see that the largest instantaneous Lyapunov exponent of the barotropic dynamics is clearly separated from the second one. That means that at any time there exists a distinct mode which maximize disturbance norm growth under the barotropic dynamics. It should be noted here that it is not a shape-preserving solutions of the dynamical equations like the familiar normal modes. These modes are eigen functions of the symmetric positive defined operator in (80) so they form a complete orthonormal basis set in the phase space of the dynamics equation. These functions have been referred as optimal singular vectors (SV) in [Molteni,Palmer,93].

The time average of the instantaneous optimal SV is presented in fig.4A. This mode is localized in space. Its maximum is always near the beginning of jet stream in the middle of basin near the Western boundary. This indicates that the largest norm of the disturbance can be obtained at the end of 1 day corresponds to the perturbation localized at the beginning of the jet stream in the middle of the basin.

Of course the optimal singular vector depends on the part of trajectory for which it is calculated. However the variability of this vector is relatively small and simple. More than $90 \%$ of this variability is concentrated in first three EOF vectors.

Contrary to fig.4A, the most unstable singular mode for the composition length 16 days is no longer localized in space. It has two clear maxima: near the Western boundary and near the Eastern one. 


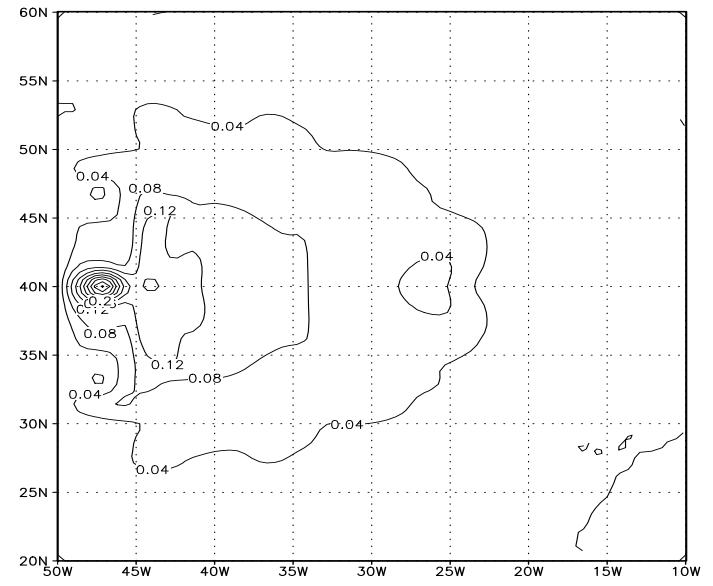

Figure 4A. The most unstable singular mode for $\mathrm{T}=1$ day.

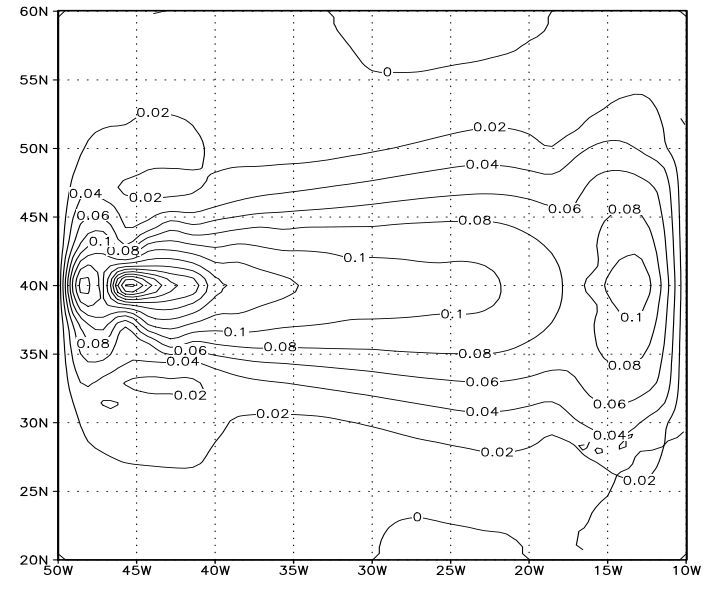

Figure 4B. The most unstable singular mode for $\mathrm{T}=16$ days.

One of the important phenomena of the finite-time predictability calculations is diminishing of local exponents with increasing of the composition length. This fact has been already noted by many authors. In [Abarbanel et al $(2), 91]$ the form of this curve is discussed for simple systems as well as the form of diminishing of the dispersion of local Lyapunov exponents. In [Dymnikov et al, 93] a discussion and an approximation of this curve has been proposed. Discussions of the finite time evolution of a small perturbation in different athmospheric and oceanic models one can find in [Lacarra,Talagrand,88], [Molteni,Palmer,93], etc.

The conclusion one can make from these discussions is that the finite time behaviour of a perturbation to the trajectoty of a nonlinear dynamical system must not be exponential.

However, calculation of Lyapunov exponents assumes implicitly that a small error grows exponentially in time. This is, of course, true for infinite time interval, but it is doubtful when we consider a limited interval. The decreasing of mean local Lyapunov exponents with increasing of their composition length shows the difference of error growth produced by real dynamics from pure exponential.

Mean local Lyapunov exponents of the barotropic model in a square for different composition lengths are presented in fig.5A. These exponents have been calculated for composition lengths $L \tau$ where $L$ is the integer power of $2: 0 \leq L \leq 11$ and $\tau=1$ day. So the interval of 2048 days has been divided into $2^{11-L}$ subintervals of $2^{L}$ days each. Local exponents of composition length $2^{L}$ days have been calculated for each subinterval and have been averaged over all subintervals. 


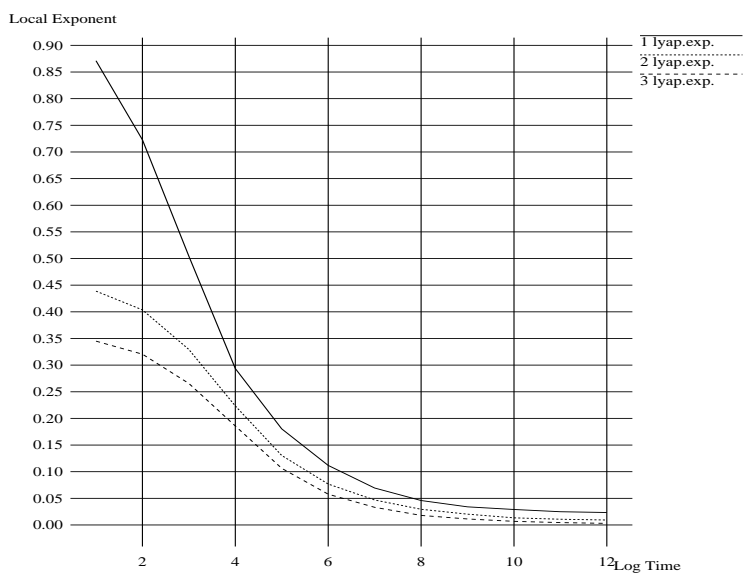

Figure 5A. Mean local Lyapunov exponents. Limit values: $\lambda_{1}^{\infty}=0.020, \lambda_{2}^{\infty}=0.0065, \lambda_{3}^{\infty}=0.001$.

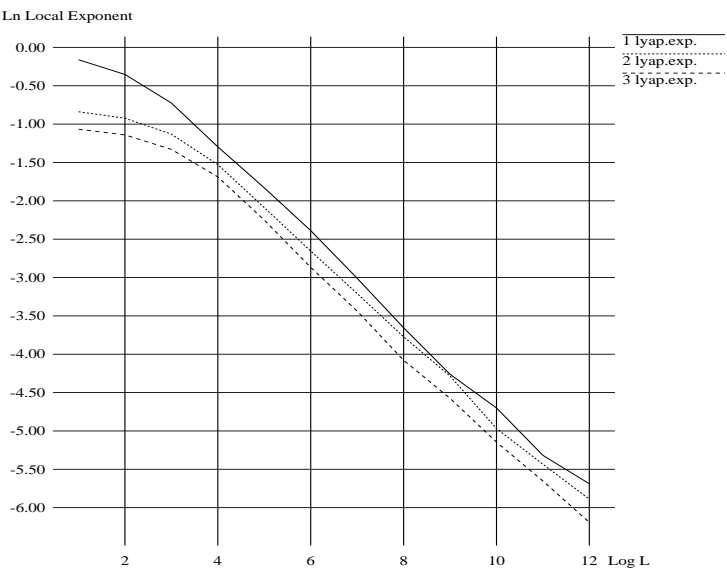

Figure 5B. Mean local Lyapunov exponents logarithms.

To evaluate the forme of the curve in fig.5A we plot the logarithm of the difference between mean local exponent of composition length $L: \overline{\lambda_{i}^{(L)}}$ and global exponent $\lambda_{i}^{\infty}$, i.e. the limit of $\overline{\lambda_{i}^{(L)}}$ when $L \rightarrow \infty: \ln \left(\overline{\lambda_{i}^{(L)}}-\lambda_{i}^{\infty}\right)$. As one can see in fig.5B this dependence is close to the linear one in logarithmic coordinates:

$$
\ln \left(\overline{\lambda_{i}^{(L)}}-\lambda_{i}^{\infty}\right) \sim A \log _{2} L+B_{i}
$$

The angular coefficient of the curves in fig.5B is about $A=-0.56$. This indicates that for $L$ sufficiently large

$$
\left.\overline{\left(\lambda_{i}^{(L)}\right.}-\lambda_{i}^{\infty}\right) \sim C_{i} \exp \left(\frac{A \ln L}{\ln 2}\right)=\frac{C_{i}}{L^{0.81}} .
$$

One can note also that three lines in the fig.5B are parallel to each other, so the coefficient $A$ does not depend on the exponent number $i$.

On these pictures one can see a rigorous decreasing of mean Lyapunov exponents when the compositon length grow. The difference between mean instantaneous exponents and global ones is about 50 times. If we assume a pure exponential growth of a perturbation, the e-folding time is about 50 days for an infinite time evolution of an error, while considering short time evolution of this error we must take into account that its e-folding time is of order of 1 day.

This fact reveals that the non-exponential growth of a small perturbation is very important for this dynamics at finite time scales.

\subsection{One layer model in a square. Higher resolution}

We shall test the numerical features of the predictability estimates algorithm. One of the principal questions here is the spatial resolution of the approximation grid. Despite there exists finite-dimensional attractor of the model (1) ([Bernier,95]) and appropriate theorems about possibility to consider it by Galerkin method ([Bernier, 90$])$, theoretical estimates of its dimension exeed considerably the number of degrees of freedom we work with. So we can not state that $\mathrm{RR} \mathrm{n}^{\circ} 3074$ 
the attractor of our finite dimensional system is close to the attractor of the model (1). To obtain an idea about the algorithm accuracy and to distinguish the most sensitive parameters we perform a numerical verification of the algorithm.

For this purpose we perform the experiment with one layer model in a square with the same parameters as above but on a finer resolution grid composed of 309 integration points. This grid has been prepared by the use of the MODULEF package also. Appropriate refining of the triangulation has been required near the western boundary and especially in the middle of the square. The grid step for this grid is about $\sqrt{2}$ times lower than for the grid presented in fig. $1 \mathrm{~A}$, so for the length of basin of $4000 \mathrm{~km}$, this value varies between $75 \mathrm{~km}$ near the Western boundary and $400 \mathrm{~km}$ near the Eastern one.

The QG model in this experiment has also been integrated during 20 years from zero state. After the spin-up the model is integrated during 2048 days with the time step 0.1 day. Average streamfunction in this experiment is shown in fig.2B.

The distributions $P\left(\lambda_{i}^{L}\right)$ of values of 3 largest Lyapunov exponents calculated in this experiment are presented in fig.6. As well as in the fig.3, this distribution is computed by sampling the 2048 daily values for the fig. $6 \mathrm{~A}$ and by sampling 128 subintervals of 16 days composition length each for the fig.6B.
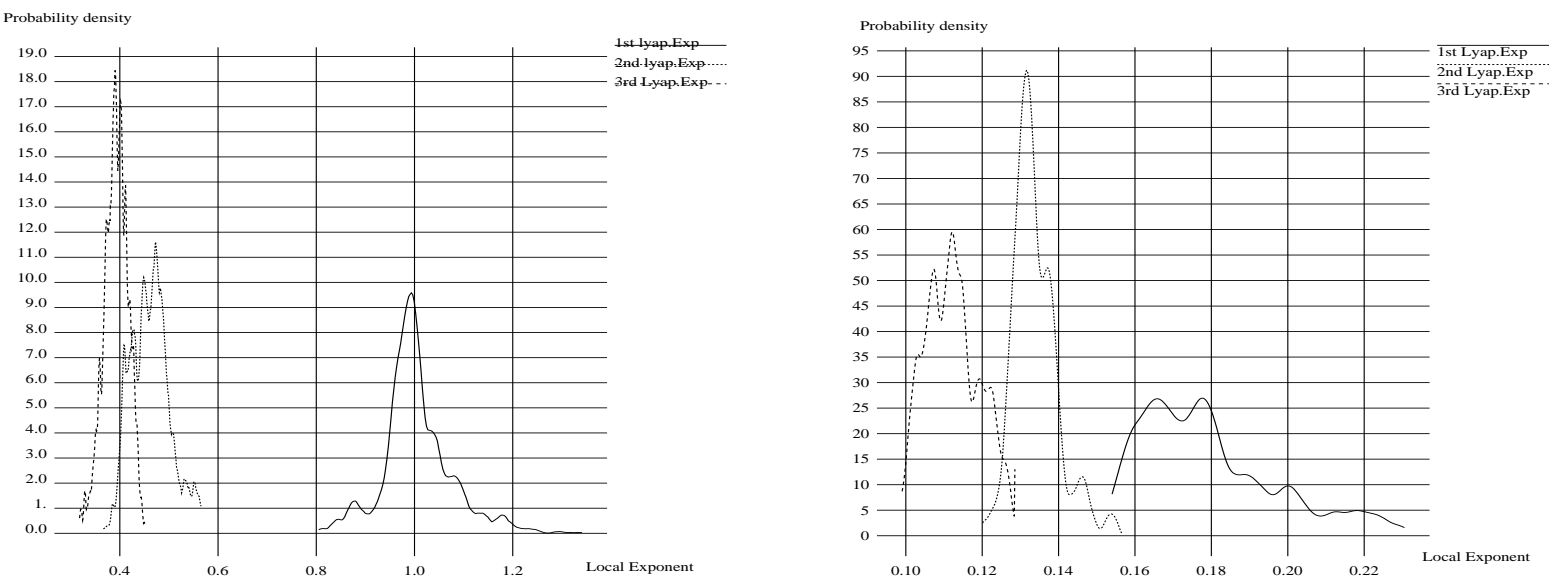

Figure 6A. Distributions of Instantaneous Lyapunov Exponents $(L=1)$.

Mean values: $\lambda_{1}=1.01, \lambda_{2}=0.46, \lambda_{3}=0.39$

Dispersions: $\sigma_{1}=0.072, \sigma_{2}=0.038, \sigma_{3}=0.022$.
Figure 6B. Distributions of local lyapunov exponents for $L \tau=16$ days.

Mean values: $\lambda_{1}=0.180, \lambda_{2}=0.134, \lambda_{3}=0.112$

Dispersions: $\sigma_{1}=0.019, \sigma_{2}=0.006, \sigma_{3}=0.007$.

Comparing fig.6 with fig. 3 we obtain that refining of the triangulation slightly enlarge the mean values. This increasing reaches $13 \%$ for the largest instantaneous exponent. Along with augmentation of the mean values one can note the change of the form of distributions. The separation of the largest exponent from others becomes more clear. Due to refining of triangulation its dispersion diminishes and the form of the distribution becomes sharper.

This exponent corresponds also to the SV localized in the region of the beginning of the jet stream. This vector is shown in fig.7A. 
The impact of the refining of the triangulation on local exponents of the composition length $L \tau=16$ days is more complex. In spite of the fact that their mean values remain almost the same, the form of distributions changes considerably: fig.3B, fig.6B.

Nevertheless the corresponding singular vector has qualitatively the same structure as in the experiment with low resolution. It has two distinct maxima near the Eastern and near Western walls (fig.7B).

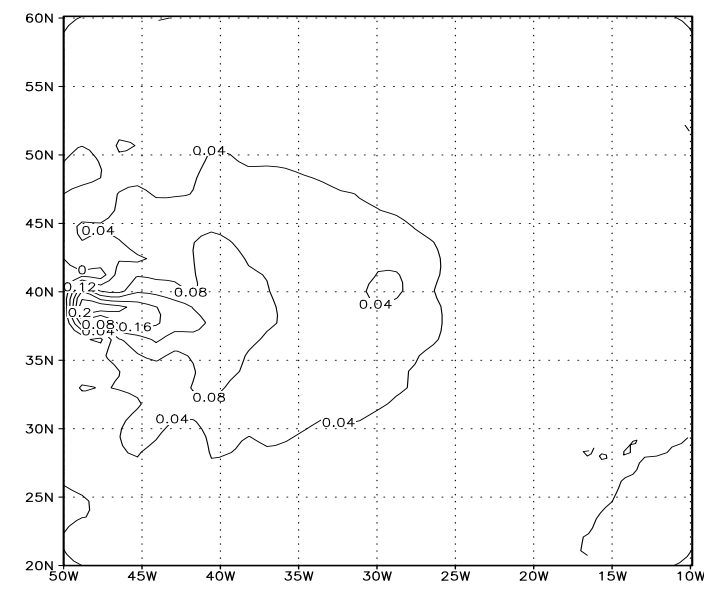

Figure 7A. The most unstable singular mode for $\mathrm{T}=1$ days.

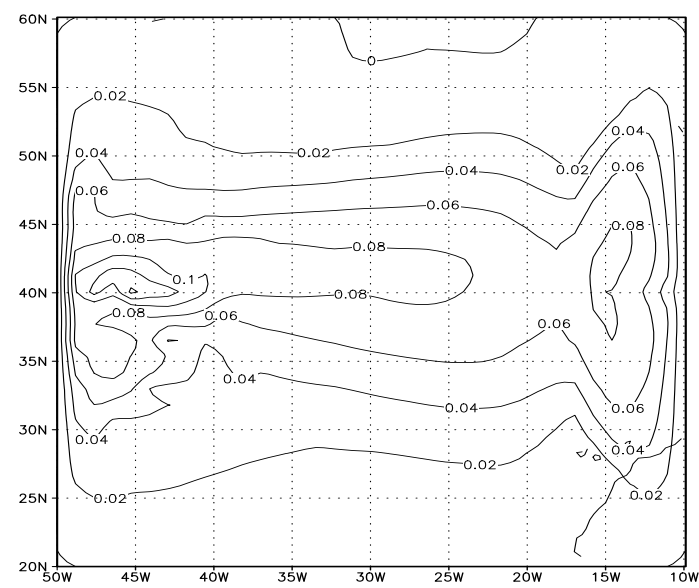

Figure 7B. The most unstable singular mode for $\mathrm{T}=16$ days.

Mean local exponents of the barotropic model in a square for finer resolution are presented in fig.8A. As well as in the fig.5A these exponents have been calculated for composition lengths $L \tau$ where $L$ is an integer power of $2: 0 \leq L \leq 11$ and $\tau=1$ day.

On fig.8A one can see that the difference between the mean instantaneous and global exponents is more rigorous than in experiment with lower resolution. That means that the nonexponential error growth for finite time scale is more important.

The form of decreasing of mean local exponents with composition length in this experiment is similar to the form of decreasing in the previous experiment. The angular coefficient of lines approximating the curves in fig. $8 \mathrm{~B}$ is equal to $A=-0.6$, so the decreasing of mean local exponents can be expressed as

$$
\left.\overline{\left(\lambda_{i}^{(L)}\right.}-\lambda_{i}^{\infty}\right) \sim C_{i} \exp \left(\frac{A \ln L}{\ln 2}\right)=\frac{C_{i}}{L^{0.87}} .
$$




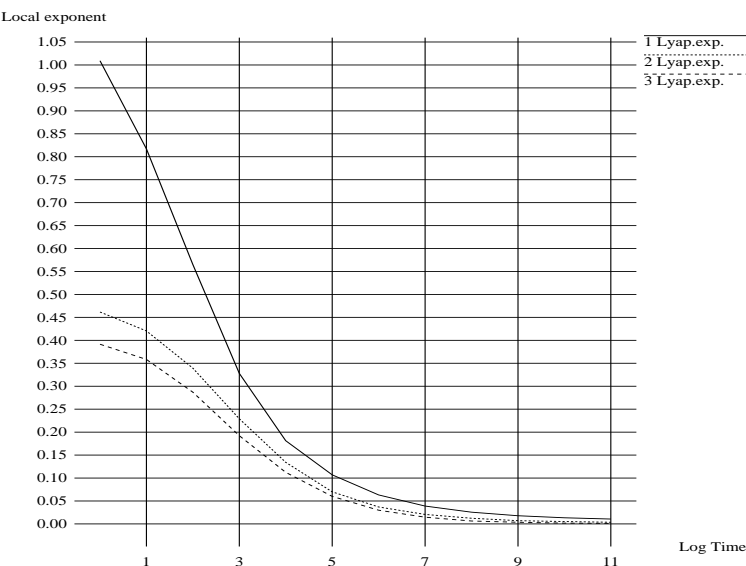

Figure 8A. Mean Local Lyapunov exponents. Limit values: $\lambda_{1}^{\infty}=0.008, \lambda_{2}^{\infty}=0.002, \lambda_{3}^{\infty}=0.0005$.

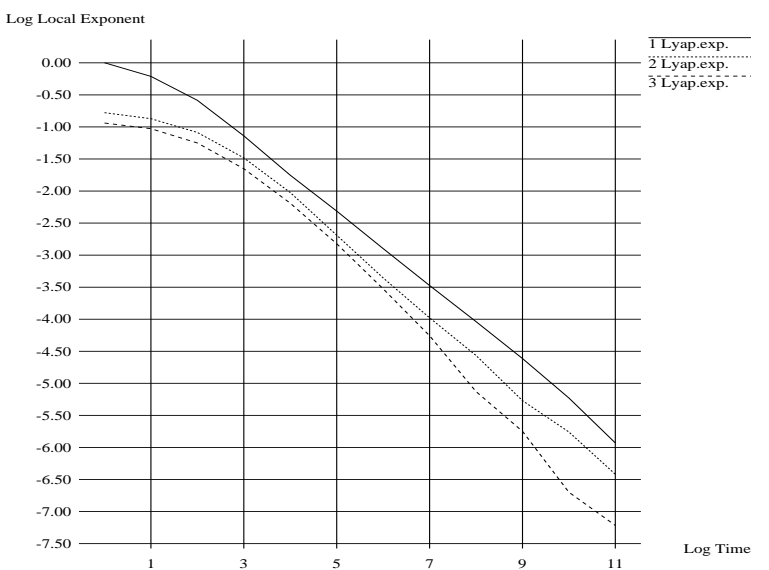

Figure 8B. Mean Local Lyapunov exponent logarithms.

\subsection{Two layer model.}

This experiment includes the modelisation of the vertical structure of the basin. We suppose that the depth of the ocean is composed by two layers of different dencity. The depth thickness of layers are equal to $1000 \mathrm{~m}$ for the surface layer, and $4000 \mathrm{~m}$ for the bottom one. The difference of the water dencity between the upper and the bottom layers is taken $\rho_{k}-\rho_{k-1}=2 \times 10^{-3} \rho_{0}$, where $\rho_{0}$ is the mean density of water. This corresponds to the reduced gravity value $g^{\prime}=0.02 \frac{\mathrm{m}}{\mathrm{s}^{2}}$ and the Rossby radius of the baroclinic mode about $55 \mathrm{~km}$.

The wind tension and dissipation parameters for this experiment have been taken the same as in two experiments above:

- forcing: $F_{\text {wind }}=-\tau_{0} \sin \frac{2 \pi y}{L}, \tau_{0}=10^{-13} s^{-2}$,

- bottom drag coefficient: $\sigma=5 \times 10^{-8} s^{-1}$,

- lateral friction: $A=2300 \frac{m^{2}}{s}$.

The QG model has been integrated during 20 years from zero state. After the spin-up the model is integrated during 2048 days with time step 0.05 day. The average streamfunction on surface and bottom layers are presented in fig.9 A and B respectively. 


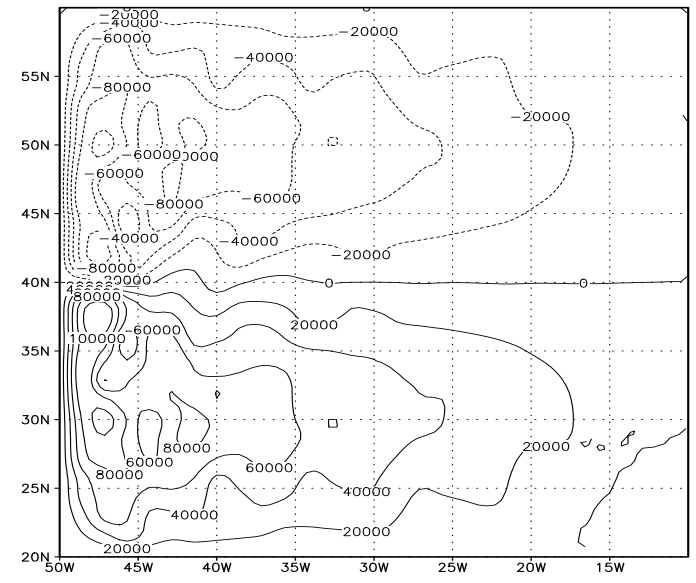

Figure 9A. Time mean streamfunction on the surface layer.

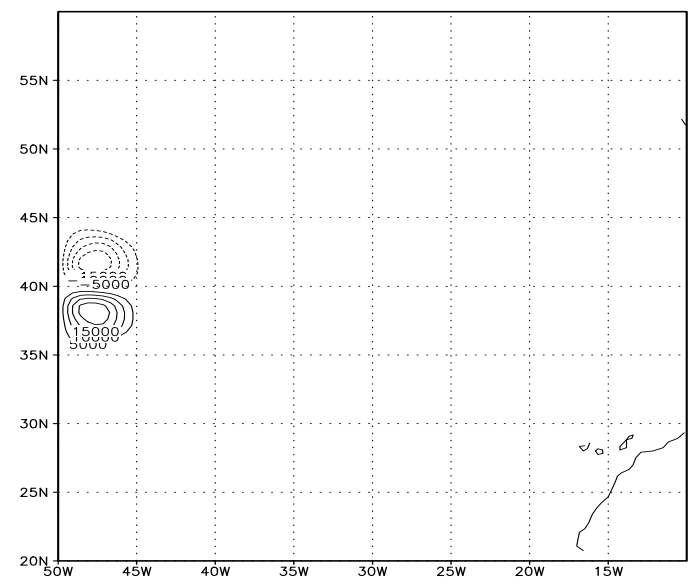

Figure 9B. Time mean streamfunction on the bottom layer.

In the fig.10A one can see the distributions of 3 largest instantaneous Lyapunov exponents computed by sampling 4096 12-hours values. In fig.10B the distributions of local Lyapunov exponents calculated by sampling of local exponents calculated for 128 subintervals of 16 days each are presented.

As well as in the experiments with barotropic model the distribution of the largest instantaneous exponent is clearly separated from others, i.e. there exists a distinct mode which maximize the error norm growth for a short time evolution. However it is not the case when we consider the error evolution during 16 days: distributions of the first and the second exponents intersect. And, as one can see in fig.12A, they tends to the same value when composition length goes to infinity.

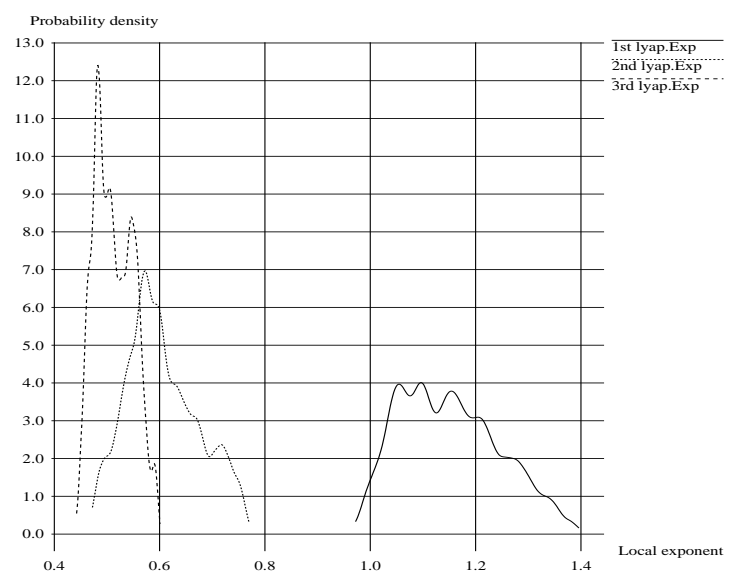

Figure 10A. Distributions of instantaneous Lyapunov Exponents $(L=1)$.

Mean values: $\lambda_{1}=1.15, \lambda_{2}=0.60, \lambda_{3}=0.51$

Dispersions: $\sigma_{1}=0.095, \sigma_{2}=0.069, \sigma_{3}=0.036$.

RR $n^{\circ} 3074$

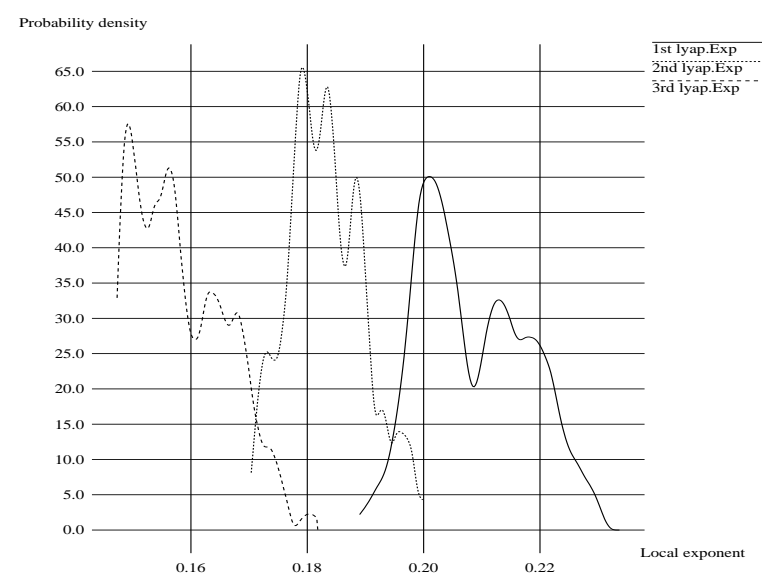

Figure 10B. Distributions of local Lyapunov exponents for $L \tau=16$ days.

Mean values $\lambda_{1}=0.209, \lambda_{2}=0.183, \lambda_{3}=0.158$.

Dispersions: $\sigma_{1}=0.009, \sigma_{2}=0.007, \sigma_{3}=0.008$. 
Instead of calculation of the most unstable singular vectors on each layer in this experiment we develop them by the system of eigenvectors of the operator $\mathcal{W}(6)$. This is a well known representation of solutions of multilayer models allowing us to consider the components corresponding to different eigenvalues of the operator $\mathcal{W}$. The passage matrix to the eigenbasis of the $\mathcal{W}$ is the matrix $\mathcal{B}(7)$.

The component corresponding to the zero eigenvalue $\Lambda_{1}=0$ of $\mathcal{W}$ we shall call the barotropic component, the other one will be named the baroclinic one.

To distinguish the barotropic and baroclinic components of a singular vector we consider their weights $\kappa_{k}$ in each vector where $k$ indicate the barotropic $(k=1)$ or baroclinic $(k=2)$ components. So far these vectors are normalized by unit:

$$
\sum_{k=1}^{2} \int_{\Omega} \Theta_{k}^{2} d \Omega=\sum_{k=1}^{2}\left(\mathcal{M} \Theta_{k}, \Theta_{k}\right)_{L_{2}}=1,
$$

we shall use $\kappa_{k}=\left(\mathcal{M} \Theta_{k}, \Theta_{k}\right)_{L_{2}}$ as the weight of $k$-th component in the singular vector. Being comprised between 0 and 1 these coefficients show us the fraction of the norm of the vector associated with its $k-t h$ component.

Barotropic components of the singular modes corresponding to the largest local exponents for the composition length 1 and 16 days are presented in fig.11A,B respectively. The weight of this component in the vector of 1 day composition length is about $80 \%$. But the singular vector becomes more and more "barotropic" when the composition length augments: the weight of this component in the 16 days vector reachs $95 \%$.

The baroclinic component (not shown) is strongly localised in the region near the beginning of the jet stream for any composition length, but its amplitude becomes negligible for the composition length 16 days.

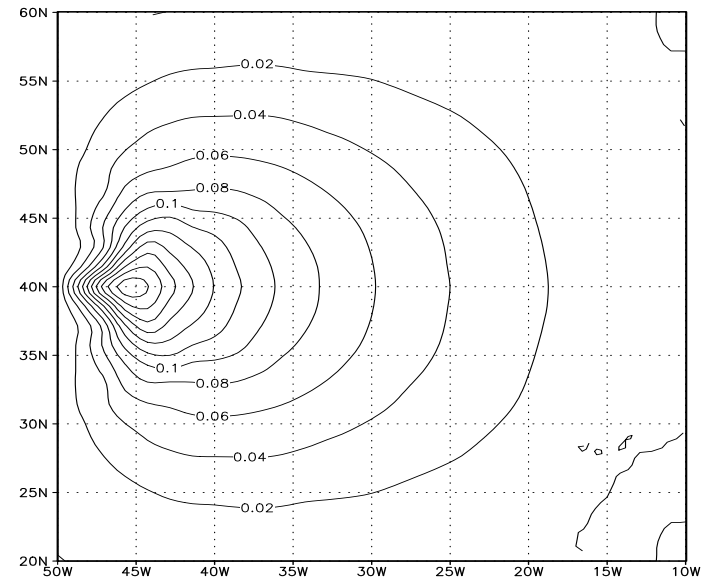

Figure 11A. The most unstable mode for $\mathrm{T}=1$ day. Barotropic component.

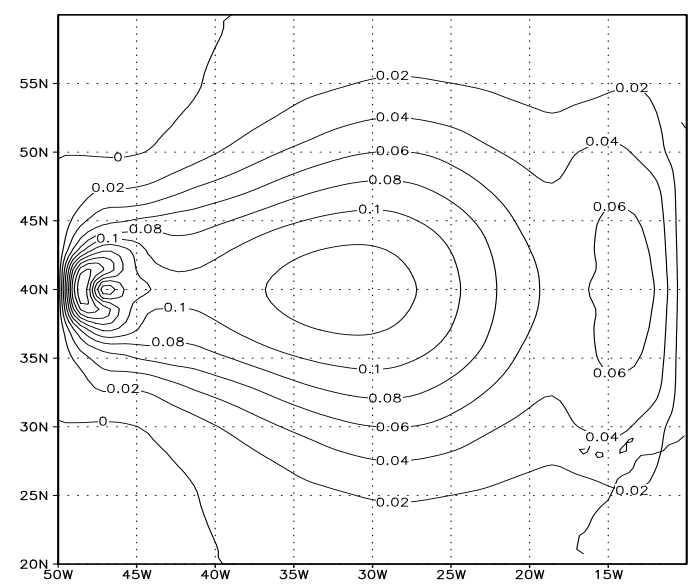

Figure 11B. The most unstable mode for $\mathrm{T}=16$ day. Barotropic component.

The dependence of the mean local exponents on the composition length in this experiment is presented in fig.12A. The particularity of this case is that two largest local exponents have the same limit when $L \tau$ tend to infinity. 
The form of decreasing of these exponents is more sharp than in previous experiments: the angular coefficient of approximating line of curves in fig.12B are equal to 0.75 for the first exponent and to 0.85 for the second and third ones.

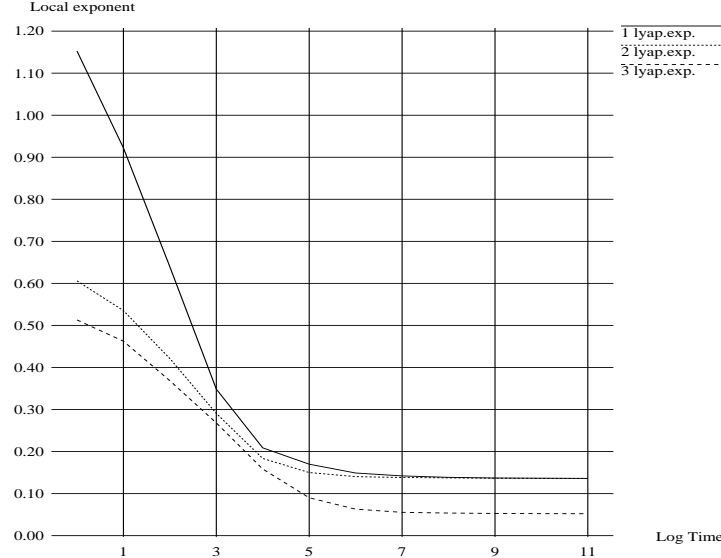

Figure 12A. Mean Local Lyapunov exponents. Limit values: $\lambda_{1}^{\infty}=0.136, \lambda_{2}^{\infty}=0.136, \lambda_{3}^{\infty}=0.052$.

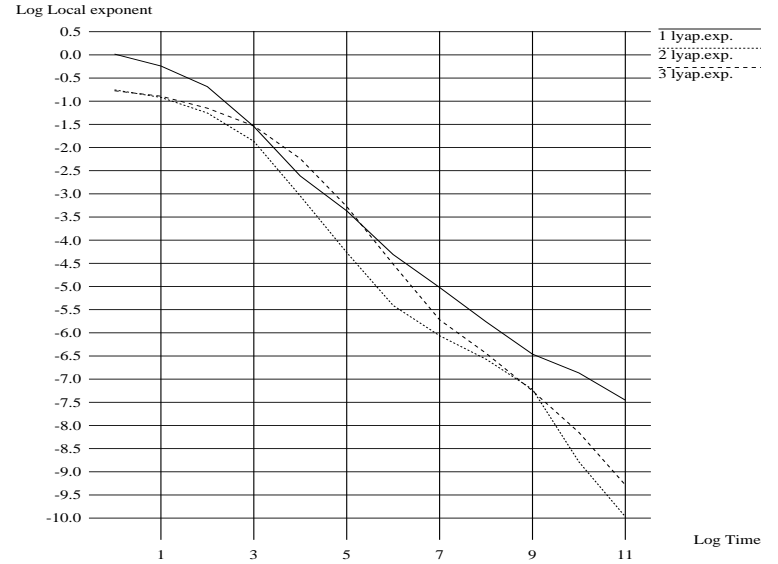

Figure 12B. Mean Local Lyapunov exponent logarithms.

\subsection{One layer model in the North Atlantic.}

To study the influence of the form of the basin on the predictability characteristics of a solution we perform the experiment in the domain which approximates the North Atlantic region. We assume that the domain is comprised in the rectangular between $78^{0} \mathrm{~W} \ldots 3^{0} \mathrm{~W}$ in longitude and $15^{0} N \ldots 65^{0} \mathrm{~N}$ in latitude. The boundary of the basin corresponds to the $1 \mathrm{kh}$ depth isobath of the ocean. The form of the domain and its triangulation is presented in fig.1B.

To obtain the forcing in this experiment we have used the data set "Monthly Global Ocean Wind Stress Components" prepared and maintained by the Data Support Section, Scientific Computing Division, National Center for Atmospheric Research. These data have been prepared by the routine described in [Hellermann, Rosenstein, 83]. From this data set we choose the mean January wind stress components $\tau_{x}$ and $\tau_{y}$ over the North Atlantic based on 1870-1976 surface observations. These data are presented on the $2^{0} \times 2^{0}$ grid.

The forcing in the equation (4) in this experiment is calculated from these data as

$$
\mathcal{F}(x, y)=-\frac{\partial \tau_{x}}{\partial \varphi}+\frac{1}{\cos \varphi} \frac{\partial \tau_{y}}{\partial \lambda}, \quad \varphi=20^{0}+y \times 50^{\circ} / L, \lambda=-40^{0}+\frac{x \times 50^{0} / L}{\cos \varphi}
$$

where $L=5500 \mathrm{~km}$ is a characteristic length of the basin. The spatial configuration of the forcing is presented in fig.13B.

The coefficient of Ekman dissipation we choose as $\sigma=1 \times 10^{-7} s^{-1}$, which corresponds to the damping timescale $T_{\sigma}=1 \times 10^{7} s \sim 100$ days. The lateral friction coefficient $A$ has been chosen in order to avoid numerical instability which occurs due to the concentration of variability of the model at grid scales. This value has been taken to be $A=2900 \frac{m^{2}}{s}$, that corresponds to the damping time scale $T_{A}=0.64$ day for a wave of $100 \mathrm{~km}$ length.

$\mathrm{RR} \mathrm{n}^{\circ} 3074$ 
As well as in previous experiments, the model has been integrated during 20 years from zero state. After the spin-up the model is integrated during 2048 days with the time step 0.05 day. This part of trajectory has been used as $\bar{\psi}(x, y, t)$ in $(70)$ to calculate local Lyapunov exponents of the model. Average streamfunction is shown in fig.13A.

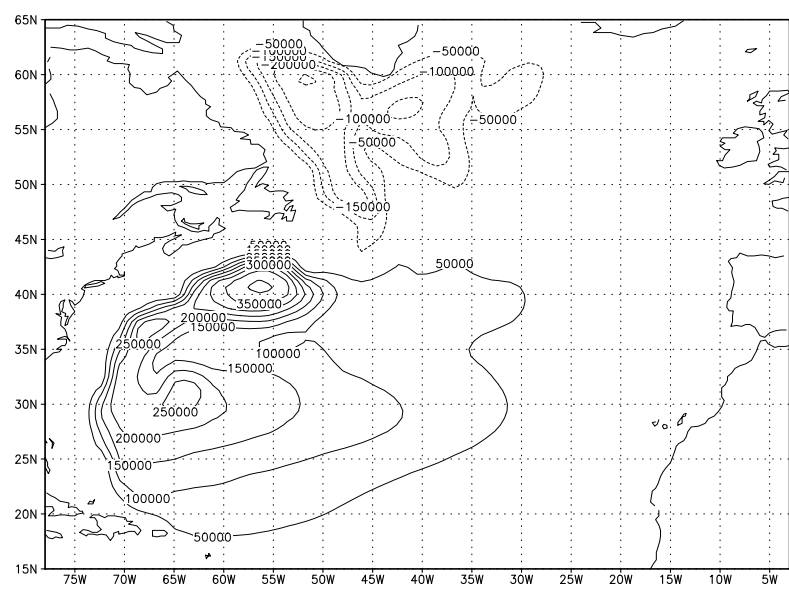

Figure 13A. Time mean streamfunction.

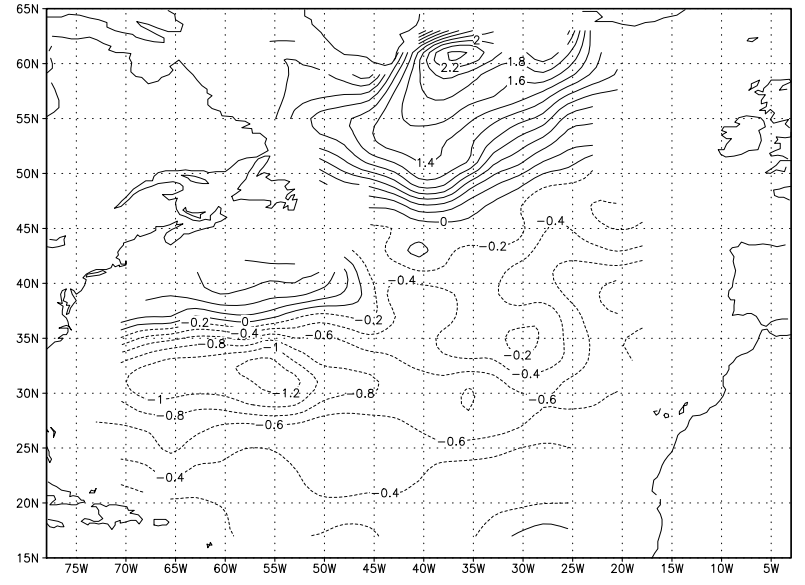

Figure 13B. Forcing of the model.

The distributions $P\left(\lambda_{i}^{L}\right)$ of values of the 3 largest Lyapunov exponents calculated in this experiment are presented in fig.14A. As well as in all previous experiments, this distribution is computed by sampling the 2048 daily values for the fig.14A and by sampling 128 subintervals of 16 days composition length each for the fig.14B.

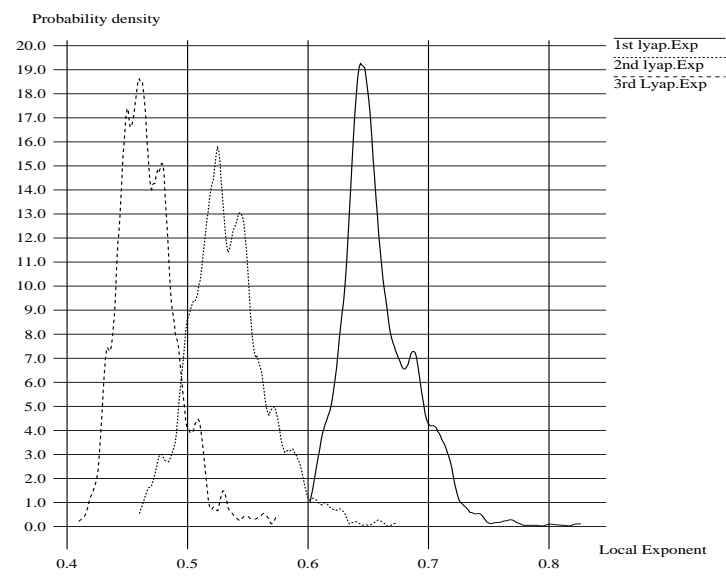

Figure 14A. Distributions of instantaneous Lyapunov Exponents $(L=1)$.

Mean values: $\lambda_{1}=0.65, \lambda_{2}=0.53, \lambda_{3}=0.46$

Dispersions: $\sigma_{1}=0.030, \sigma_{2}=0.032, \sigma_{3}=0.025$.

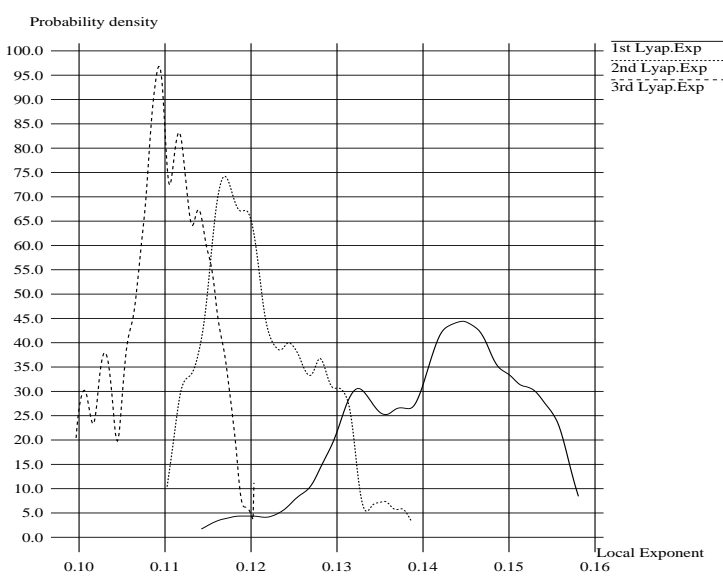

Figure 14B. Distributions of local Lyapunov exponents for $L \tau=16$ days.

Mean values $\lambda_{1}=0.142, \lambda_{2}=0.121, \lambda_{3}=0.110$

Dispersions: $\sigma_{1}=0.009, \sigma_{2}=0.006, \sigma_{3}=0.005$. 
The time average of the instantaneous optimal singular vector is presented in fig.15A. Despite that at any time this mode is localized in space, its time mean is not localised. Contrary to previous experiments in a square, there is no distinct place of localisation of this mode. Its maximum is situated near the near the American coast but at different latitudes.

The variability of this vector becomes more complecs with respect to experiments in a square. The first three EOF vectors concentrate about $60 \%$ of variability only.

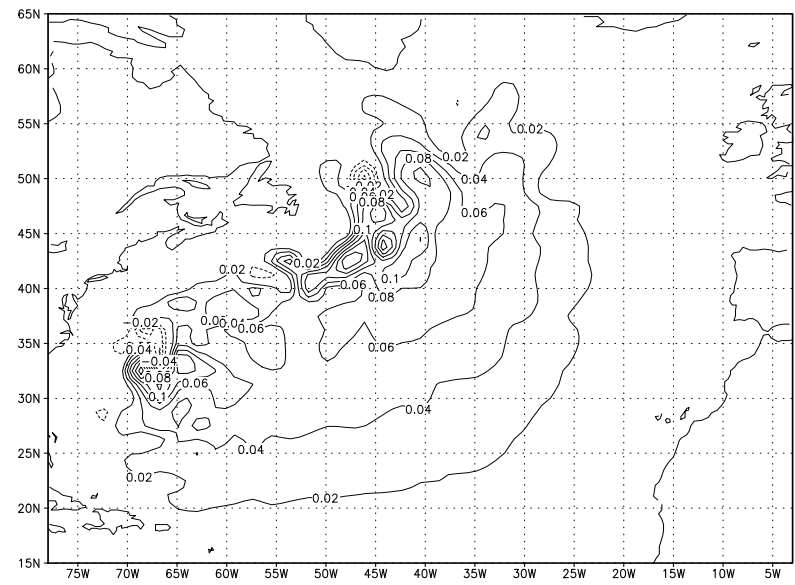

Figure 15A. The most unstable mode for $\mathrm{T}=1$ day.

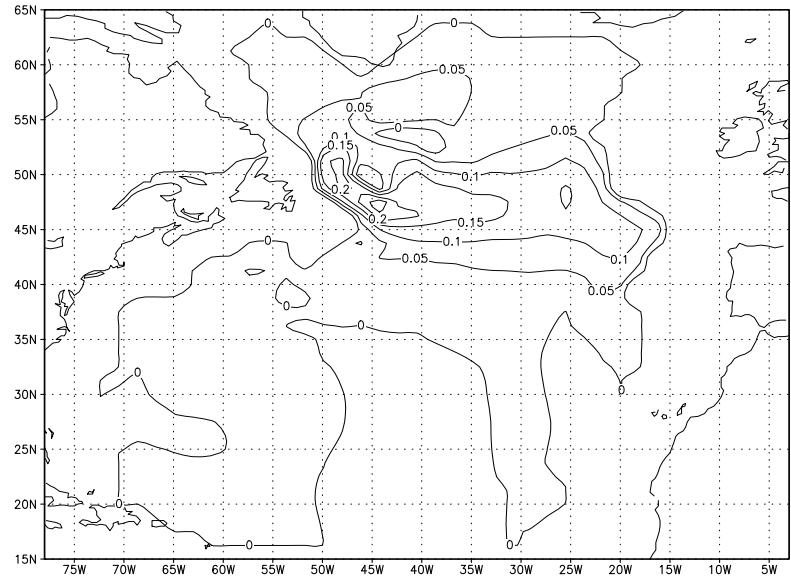

Figure 15B. The most unstable mode for $\mathrm{T}=16$ day.

Mean local Lyapunov exponents of the barotropic model in the North Atlantics for different composition lengths are presented in fig.16A. These exponents have been calculated for composition lengths $L \tau$ where $L$ is the integer power of 2: $0 \leq L \leq 9$ and $\tau=1$ day. So the interval of 2048 days has been divided into $2^{11-L}$ subintervals of $2^{\bar{L}}$ days each. Local exponents of composition length $2^{L}$ days have been calculated for each subinterval and have been averaged over all subintervals.

The logarithm of the difference between mean local exponent of composition length $L \overline{\lambda_{i}^{(L)}}$ and global exponent $\lambda_{i}^{\infty}$ is presented in fig.16B. As well as in previous experiments the dependence in fig. $5 \mathrm{~B}$ is also close to the linear one in logarithmic coordinates:

$$
\ln \left(\overline{\lambda_{i}^{(L)}}-\lambda_{i}^{\infty}\right) \sim A \log _{2} L+B
$$

The angular coefficient of the curves in fig.5B is about $A=-0.75$. This indicates that for $L$ sufficiently large

$$
\left.\overline{\left(\lambda_{i}^{(L)}\right.}-\lambda_{i}^{\infty}\right) \sim C_{i} \exp \left(\frac{A \ln L}{\ln 2}\right)=\frac{C_{i}}{L^{1.08}} .
$$




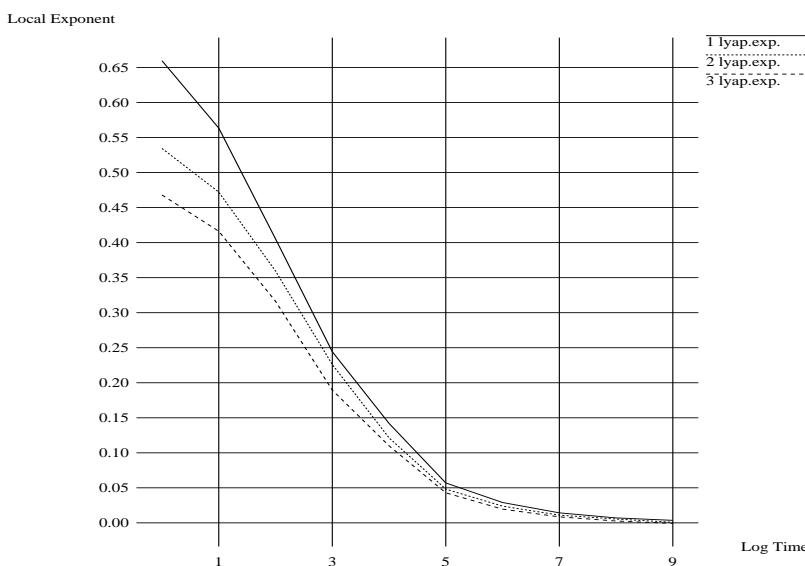

Figure 16A. Mean Local Lyapunov exponents. Limit values: $\lambda_{1}^{\infty}=8 \times 10^{-4}, \lambda_{2}^{\infty}=-1.5 \times 10^{-3}, \lambda_{3}^{\infty}=$ $-2.8 \times 10^{-3}$.

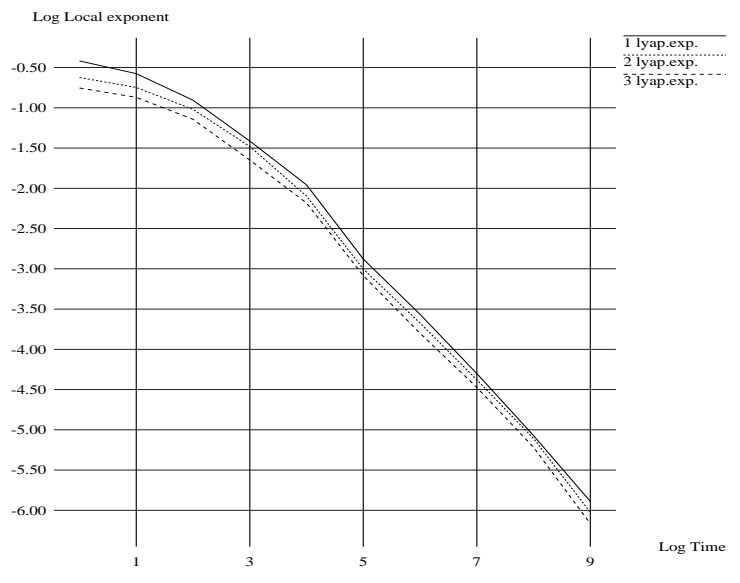

Figure 16B. Mean Local Lyapunov exponent logarithms.

\section{Conclusion}

Traditionally in the analysis of a chaotic dynamical system, the Lyapunov exponents are used to quantify the notion of predictability of the system. However, use of classical Lyapunov exponents is inadequate when a finite time predictability is addressed.

The first reason of this is well known: local variations of predictability on an attractor can lead to significant variations of the divergence rate of trajectories. So to estimate shorttime predictability one needs the local information rather than the global one. This aspect is represented in this paper by the distribution functions of the 3 largest exponents fig.3, fig.6, fig.10, fig.14. For the QG model these variations can reach $30 \%$ of the mean value.

Along with local variations of predictability, one should take into account the dependence of the local exponents on the time scale of the prediction itself due to nonexponential error growth. This kind of variation can be even more important than phase spatial variations as it takes place in this model. Averaging over attractor local exponents exhibit very large dependence on the composition length. This fact reveals the importance of consideration of the different mechanisms of trajectory divergence on finite time scales.

For many systems the decreasing of averaged local exponents is close to linear in the logarithmic coordinates. So the difference between averaged local exponents and the global ones decrease as

$$
\left.\overline{\left(\lambda_{i}^{(T)}\right.}-\lambda_{i}^{\infty}\right) \sim \frac{C_{i}}{T^{\alpha}}
$$

As we have seen, the quantities of $C_{i}$ and $\alpha$ seems to be characteristics of the attractor. Moreover, the value of $\alpha$ appears to be constant for all exponents. This could provide us with the possibility to reconstruct global exponents from the set of averaged values of the local ones. Once this set has been calculated for the $\overline{\lambda_{i}^{(T)}}$ we can obtain the asymptotic global exponent by reconstruction of $\alpha$ and extrapolation them from finite data. 
Despite local exponent values are rather sensitive to the numerical aspects, corresponding singular function reveals rather high stability under any numerical variations. The first singular function presented in this paper conserves its principal properties under variations of vertical and spatial resolution and domain geometry. Instantaneous singular vector remains concentrated near the Western boundary while the singular function calculated for the 16 days has two maxima: near the Western boundary and near the Eastern one.

Acknowledgements. This work was performed at the University Nancy I, INRIALorraine under grant of the French Ministry of High Education and Research. The author has benefited the support of the A.M. Liapunov French-Russian Institute of Computer Science and Applied Mathematics. I thank Christine Kazantsev and Michel Pierre for the very helpful discussions.

\section{References}

[Abarbanel et al(1), 91] H.D.I. Abarbanel, R. Brown, M.B. Kennel. Lyapunov exponents in chaotic systems: their importance and their evaluation using observed data. Invited review for Modern physics letters B. (1991)

[Abarbanel et al(2), 91] H.D.I. Abarbanel, R. Brown, M.B. Kennel. Variations of Lyapunov exponents on a strange attractor., J.Nonlinear Sci. vol.1 (1991).

[Bengtsson,Temperton,79] L. Bengtsson, L. Temperton. Difference approximation to quasigeostrophic models. Numerical methods used in atmospheric models. GARP publication series 17, vol. 2, WMO/ICSU Joint organizing Commitee (1979).

[Bernadou,88] M. Bernadou. Modulef: une bibliothèque modulaire d'éléments finis. INRIA (1988).

[Bernier,90] Ch. Bernier. Etude et parallélisation d'un code d'éléments finis pour la modélisation quasi-géostrophique des circulations océaniques. PhD thesis of INPG, Grenoble, France (1990).

[Bernier,94] Ch. Bernier. Existence of attractor for the quasi-geostrophic approximation of the Navier-Stokes equations and estimate of its dimension. Advances in mathematical sciences \& Applic. Vol.4, N2, pp.465-489 (1994).

[Bernier,95] Ch. Bernier Predictability of Oceanic circulations. Rapport de recherche INRIA, N $2582,(1995)$.

[Charney, De Vore, 79] J.G. Charney, J.G. De Vore. Multiple flow equilibria in the atmosphere and blocking. J.Atm.Sci. 36, p.1205-1216 (1979).

[Dymnikov et al, 90] V.P. Dymnikov, E.V. Kazantsev, V.V. Kharin. Stability measures and lifetimes of atmospheric circulation regimes. Izvestiya, Atmospheric and Oceanic Physics. Vol. 26 , No $4(1990)$

[Dymnikov et al, 92] V.P. Dymnikov, E.V. Kazantsev, V.V. Kharin. Information entropyand local Lyapunov exponents of barotropic atmospheric circulation. Izvestiya, Atmospheric and Oceanic Physics. Vol. 28, No 6 (1992).

$\mathrm{RR} \mathrm{n}^{\circ} 3074$ 
[Dymnikov et al, 93] V.P. Dymnikov, E.V. Kazantsev. On the attractor structure generated by the system of equation of the barotropic atmosphere. Izvestiya, Atmospheric and Oceanic Physics, Vol. 29, No 5 (1993).

[Fix,75] G.J. Fix. Finite elements models for ocean circulation problems. J. Appl.Math. 29, 371 (1975).

[Grassberger, Procaccia, 83] P. Grassberger, I. Procaccia. Measuring the strangeness of strange attractors. Physica D. V.90, N.1, p.189-208 (1983).

[Hellermann, Rosenstein, 83] S. Hellerman, M. Rosenstein. Normal Monthly Wind Stress over the World Ocean with Error Estimates. Journal of Phys. Oceanography. Vol. 13, No. 7, pp. 1093-1104, (1983).

[Lorenz,63] E.N. Lorenz. Deterministic nonperiodic flow. J.Atm.Sci., 20, 130-141 (1963).

[Holland,78] W.R. Holland. The role of mesoscale eddies in the general circulation of the ocean - numerical experiments using wind driven quasi-geostrophic model. Journal of physical oceanography, Vol.8, No.3 (1978).

[Il'in,91] A.A. Il'in. The Navier-Stokes and Euler equations on two-dimensional closed manifolds. Math. USSR Sbornik, Vol 69, N 1 (1991).

[Kaplan, Yorke,79] J.L. Kaplan, J.A. Yorke. Chaotic behaviour in multidimensional difference equations. Lecture notes in mathematics, No.730 (1979).

[Krishnamurty,93] V. Krishnamurty. A predictability study of Lorenz's 28-variable model as a dynamical system. J.Atm.Sci, 50, pp.2215-2229 (1993).

[Le Provost, Bernier, Blayo, 94] C. Le Provost, Ch. Bernier, E. Blayo. A comparison of two numerical methods for integrating a quasi-geostrophic multilayer model of ocean circulations: finite element and finite difference methods. Journal of computational physics, vol.110, No.2 (1994).

[Lacarra,Talagrand,88] J.-F. Lacarra, O. Talagrand. Short range evolution of small perturbation in a barotropic model. Tellus, 40A, pp.81-95 (1988).

[Legras,Ghil,85] B.Legras, M. Ghil. Persistent anomalies, blocking and variations in atmospheric predictability. J.Atm. Sci, 42, 433-471 (1985).

[Le Provost, Verron,87] C. Le Provost, J. Verron. Wind-driven ocean circulation transition to barotropic instability. Dyn.Atmos.Oceans, vol.11 (1987)

[Molteni,Palmer,93] F. Molteni, T.N. Palmer. Predictability and finite time instability of the northern winter circulation. Quarterly Journal of Royal meteorological Society. V.119, pp.269298 (1993).

[Nicolis et. al. 95] C. Nicolis, S. Vannitsem and J.-F. Royer. Short-range predictability of the atmosphere: Mechanism for superexponential error growth. Q.J.R.Meteorol. Soc., 1221, pp.705-722 (1995). 
[Oseledets,68] V.I. Oseledets. A Multiplicative Ergodic theorem. Lyapunov characteristic numbers for Dynamical Systems. Moscow Math. Soc. V.19, 197 (1968)

[Pesin,77] Ya.B. Pesin. Lyapunov characteristic exponents and smooth ergodic theory. Russian math surveys, vol. 32 (1977).

[Ruelle,90] R. Ruelle. Deterministic chaos: the science and the fiction. Proc. Roy.Soc. London. V. A427, p. 241 (1990).

[Temam,88] R. Temam. Infinite-dimensional dynamical systems in mechanics and physics. Applied Mathematical Sciences, N.68, Springer-Verlag (1988)

[Trevisan, 93] A. Trevisan. Impact of transient error growth on global average predictability measures. J.Atm.Sci., 50, pp.1016-1028 (1993).

$\operatorname{RR} n^{\circ} 3074$ 


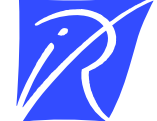

Unit ${ }^{e}$ de recherche INRIA Lorraine, Technop^ole de Nancy-Brabois, Campus scientifique, 615 rue du Jardin Botanique, BP 101, 54600 VILLERS LÈS NANCY

Unit'e de recherche INRIA Rennes, Irisa, Campus universitaire de Beaulieu, 35042 RENNES Cedex

Unit'e de recherche INRIA Rh`one-Alpes, 655, avenue de l'Europe, 38330 MONTBONNOT ST MARTIN

Unit'e de recherche INRIA Rocquencourt, Domaine de Voluceau, Rocquencourt, BP 105, 78153 LE CHESNAY Cedex

Unit'e de recherche INRIA Sophia-Antipolis, 2004 route des Lucioles, BP 93, 06902 SOPHIA-ANTIPOLIS Cedex

Éditeur

INRIA, Domaine de Voluceau, Rocquencourt, BP 105, 78153 LE CHESNAY Cedex (France)

ISSN 0249-6399 